\title{
C/EBP $\varepsilon$ mediates nicotinamide-enhanced clearance of Staphylococcus aureus in mice
}

\author{
Pierre Kyme, 1,2,3 Nils H. Thoennissen,4,5 Ching Wen Tseng, ${ }^{1,2,3}$ Gabriela B. Thoennissen, ${ }^{4,5}$ \\ Andrea J. Wolf, ${ }^{2}$ Kenichi Shimada, ${ }^{1,2,3}$ Utz O. Krug, ${ }^{5}$ Kunik Lee, ${ }^{4}$ Carsten Müller-Tidow, ${ }^{5}$ \\ Wolfgang E. Berdel, ${ }^{5}$ W. David Hardy, ${ }^{6}$ Adrian F. Gombart, ${ }^{7}$ \\ H. Phillip Koeffler, ${ }^{4,8}$ and George Y. Liu'1,2,3
}

\begin{abstract}
${ }^{1}$ Division of Pediatric Infectious Diseases and The Research Division of Immunology, Department of Biomedical Sciences, and ${ }^{2}$ Immunobiology Research Institute, Cedars-Sinai Medical Center, Los Angeles, California, USA. ${ }^{3}$ Department of Pediatrics, David Geffen School of Medicine, UCLA, Los Angeles, California, USA. ${ }^{4}$ Division of Hematology and Oncology, Cedars-Sinai Medical Center, UCLA School of Medicine, Los Angeles, California, USA. ${ }^{5}$ Department of Medicine A, Hematology, Oncology and Pneumology, University of Muenster, Muenster, Germany. ${ }^{6}$ Division of Infectious Diseases, Cedars-Sinai Medical Center, Los Angeles, California, USA. ${ }^{7}$ Linus Pauling Institute, Department of Biochemistry and Biophysics, Oregon State University, Corvallis, Oregon, USA. ${ }^{8}$ National Cancer Institute of Singapore, National University, Singapore.
\end{abstract}

\begin{abstract}
The myeloid-specific transcription factor, CCAAT/enhancer-binding protein $\varepsilon(C / E B P \varepsilon)$ is a critical mediator of myelopoiesis. Mutation of this gene is responsible for neutrophil-specific granule deficiency in humans, a condition that confers susceptibility to Staphylococcus aureus infection. We found that C/EBPE-deficient mice are severely affected by infection with $S$. aureus, and C/EBPE deficiency in neutrophils contributes to the infectious phenotype. Conversely, exposure to the epigenetic modulator nicotinamide (vitamin B3) increased expression of C/EBP $\varepsilon$ in WT myeloid cells. Further, nicotinamide increased the activity of C/EBP $\varepsilon$ and select downstream antimicrobial targets, particularly in neutrophils. In a systemic murine infection model as well as in murine and human peripheral blood, nicotinamide enhanced killing of $S$. aureus by up to 1,000 fold but had no effect when administered to either C/EBPE-deficient mice or mice depleted of neutrophils. Nicotinamide was efficacious in both prophylactic and therapeutic settings. Our findings suggest that $\mathrm{C} / \mathrm{EBP} \varepsilon$ is an important target to boost killing of bacteria by the innate immune system.
\end{abstract}

\section{Introduction}

Staphylococcus aureus in community and health care settings commonly causes serious and potentially life-threatening infections (1-3). Widespread use of antibiotics is responsible for the emergence and rapid spread of resistant pathogens, including methicillin-resistant $S$. aureus (MRSA) (3), and highlights a pressing need for development of novel antimicrobial therapies.

Increasingly, novel therapeutics are identified by studying host and bacterial factors that play important roles in the immunopathology of infection. For example, the golden pigment of $S$. aureus is an important virulence factor that shields the pathogen from host oxidative killing, and we have previously shown that blocking the biosynthesis of this pigment could be a strategy for treatment of $S$. aureus infection (4). Conversely, among human genetic conditions that alter susceptibility to $S$. aureus infection is neutrophil-specific granule deficiency (SGD), a rare hematologic disorder characterized by a significantly defective immunity (5-8). Patients with SGD present with functional defects in neutrophils, as well as monocytes/macrophages, and suffer from recurrent life-threatening bacterial infections, including S. aureus, Pseudomonas aeruginosa, and Klebsiella pneumoniae. Therefore, understanding the defective host immune mechanisms conferring susceptibility to $S$. aureus could lead to identification of immune modulatory strategies (9).

Authorship note: Pierre Kyme and Nils H. Thoennissen contributed equally to this work and are co-first authors. H. Phillip Koeffler and George Y. Liu contributed equally to this work and are co-senior authors.

Conflict of interest: The authors have declared that no conflict of interest exists. Citation for this article: J Clin Invest. 2012;122(9):3316-3329. doi:10.1172/JCI62070.
Recently, we and others have established the essential role of the myeloid-specific transcription factor CCAAT/enhancer-binding protein $\varepsilon(\mathrm{C} / \mathrm{EBP} \varepsilon)$ in the terminal differentiation as well as the functional maturation of neutrophils and monocytes/macrophages (10-17). Neutrophils from C/EBPE-deficient mice (Cebpe ${ }^{-/}$ mice) display aberrant phagocytosis, respiratory burst, and bactericidal activities. This phenotype is similar to that of neutrophils from individuals with SGD, which led to the discovery of germline loss-of-function mutations involving CEBPE in individuals suffering from this disease $(11-15,18,19)$. Importantly, in the presence of all other C/EBP family members, Cebpe $e^{-/-}$neutrophils lack expression of all secondary (specific) granule proteins, including important antimicrobials, such as lactoferrin (LTF), cathelicidin antimicrobial peptide (CAMP), neutrophil gelatinase (NGAL), and neutrophil collagenase. In particular, LTF and CAMP have been shown to exhibit anti-S. aureus activity (20-22). In addition, murine and human monocytes/macrophages with impaired expression of C/EBPE display signs of immaturity, impaired phagocytosis, and altered myelomonocytic-specific gene expres$\operatorname{sion}(8,13,16,17)$.

Genome-wide expression analyses revealed a substantial role for histone deacetylases (HDACs) in the regulation of host defense genes, including complement factors, cytokines, chemokines, and transcriptional regulators (23-27). Also, the activity of the highly conserved family member $\mathrm{C} / \mathrm{EBP} \beta$ is regulated in part by its acetylation and deacetylation (28). Therefore, modification of acetylation could be important for the regulation of the transcription factor C/EBPE and its downstream antimicrobial targets. HDAC inhibitors are essential epigenetic regulators of transcription that modify acetylation of histones and nonhistone proteins (23-27). 
These inhibitors can block the activity of certain HDACs and induce histone acetylation, leading to the relaxation of chromatin structure with enhanced accessibility of transcriptional machinery to DNA and increased gene transcription (23-25). HDAC inhibitors may also induce protein acetylation of transcription factors, resulting in changes in their transcriptional activity and of downstream target genes $(26,27)$. Nicotinamide (NAM), also referred to as vitamin B3, is the amide of nicotinic acid and is well known to act as a competitive inhibitor of class III HDACs (29-31).

The role of NAM as a modulator of inflammation has been widely reported. For example, NAM is prescribed topically for treatment of a number of inflammatory skin conditions, including acne vulgaris and atopic dermatitis (32). In experimental murine models of Gram-positive and Gram-negative sepsis, NAM has been shown to improve survival $(33,34)$. The mechanism contributing to immune modulation is not well-defined, but NAM has also been shown to suppress secretion of a number of cytokines and chemokines, including IL-1 $\beta$, TNF- $\alpha$, IL-6, and iNOS $(35,36)$. Cellular targets of NAM include protein kinase $\mathrm{B}$, forkhead transcription factors, poly(ADP-ribose) polymerase, and cysteine proteases (37). Suppression of the nuclear enzyme poly(ADP-ribose) polymerase is thought to be potentially important $(38,39)$, as it contributes to tissue injury by depletion of $\mathrm{NAD}^{+}$and by upregulation of inflammatory cytokines and chemokines $(40,41)$. More recently, NAM has been shown to induce granulocytosis in human subjects by activation of C/EBP $\alpha$ and G-CSF (42).

Interestingly, despite its implication in a number of host responses, the role of NAM as an antimicrobial agent is limited. NAM has been shown to be effective in amelioration of Mycobacterium tuberculosis disease in human subjects (43). More recently, Wurtele and colleagues reported that modulation of the yeast histone H3 Lys56 acetylation by NAM sensitized Candida albicans to genotoxic and antifungal agents (44).

Though Cebpe $e^{-/}$mice appear more susceptible to spontaneous bacterial infection during their life span (12), in vivo challenge experiments with a bacterial pathogen have not been conducted in the $\mathrm{C} / \mathrm{EBP} \varepsilon$-deficient background. In this study, we investigated the critical role of C/EBPE in the innate immune response against $S$. aureus. We showed that, in the absence of functional C/EBPE, mice were severely impaired in their ability to clear $S$. aureus infection. Neutrophils from Cebpe $e^{-/-}$mice were unable to kill S. aureus and contributed to the pathophysiology of infection. Because C/EBPE appeared essential for defense against $S$. aureus, we hypothesized that a pharmacologic agent that enhanced expression of C/EBPE above physiologic level might lead to therapeutic killing of $S$. aureus. In this study, we showed that NAM could increase C/EBPE activity and that this in turn led to effective immune-mediated clearance of $S$. aureus.

\section{Results}

Impaired response of Cebpe ${ }^{-/-}$mice to $S$. aureus infection. Humans and mice without functional C/EBPE have significant neutrophil and monocyte/macrophage defects, comparable to those of individuals with SGD (11-19). To determine the critical role of C/EBPE in $S$. aureus infection, we challenged WT and Cebpe $e^{-/-}$mice with different doses of S. aureus s.c. Compared with WT mice, Cebpe $e^{-/}$mice exhibited dramatic weight loss, significantly larger skin lesion size, and more CFUs in the lesions (Figure 1, A-C, and Supplemental Figure 1). Additionally, s.c. infection of Cebpe $e^{-/-}$mice was associated with increased systemic spread of bacteria to the spleens and kidneys on day 6 after infection (p.i.; Figure 1C).
Histopathological evaluation with $\mathrm{H} \& \mathrm{E}$ revealed a significantly larger number of neutrophils and macrophages in the skin lesions of Cebpe $e^{-/-}$mice 24 hours p.i. compared with that in WT mice (Figure 1D and Supplemental Figure 2; supplemental material available online with this article; doi:10.1172/JCI62070DS1), which was accompanied by high levels of the chemokines CXCL1 (also known as KC) and CXCL2 (also known as MIP2) (Figure 1E). These data suggest that enhanced accumulation of phagocytic cells at the infection site in Cebpe $e^{-/-}$mice failed to control S. aureus infection and point to the severe defects in the antimicrobial machinery in Cebpe $e^{-/-}$phagocytic cells.

To formally assess the ability of Cebpe $e^{-/-}$phagocytic cells to kill S. aureus, we used a well-described phagocytic survival assay, in which peripheral blood from WT and Cebpe $e^{-/}$mice was infected with $S$. aureus ex vivo. The assay is commonly used as an approximate measure of neutrophil killing function. As anticipated, bacterial clearance was significantly decreased in the blood of the Cebpe $e^{-/-}$mice compared with that of WT mice (Figure 1F and Supplemental Figure 3). By comparison, overall bacterial clearance by Cebpe $e^{-/-}$bone marrow-derived macrophages (BMDMs) was not significantly different compared with clearance by WT macrophages (Figure 1G).

Neutrophils are a critical component of the host immune response against $S$. aureus. To determine the contribution of $\mathrm{Cebpe}^{-/-}$neutrophils toward infection in vivo, we performed infection experiments in WT and Cebpe $e^{-/}$mice depleted of neutrophils. Depletion was achieved by daily injection of mice with a murine anti-polymorphonuclear neutrophil (anti-PMN) antibody, starting 24 hours prior to s.c. infection with S. aureus. Administration of anti-PMN antibody had no depleting effect on the CD11b-positive, Ly6G-negative population (monocytes/macrophages), based on analysis of splenocytes by flow cytometry (data not shown). As expected, in the absence of neutrophils, WT mice showed more CFUs at the site of infection (Figure $1 \mathrm{H}$ ). By contrast, Cebpe $e^{-/}$mice depleted of neutrophils showed significantly fewer CFUs within the lesion compared with Cebpe $e^{-/}$mice with neutrophils (Figure $1 \mathrm{H}$ ), suggesting that $\mathrm{C} / \mathrm{EBP} \varepsilon$ deficiency in neutrophils is a major contributor to the severity of infection in the knockout mice. We corroborated this finding using a gentamicin protection assay (intracellular survival assay) performed on WT and Cebpe $e^{-/-}$whole blood. We showed that $S$. aureus was readily cleared from WT blood but persisted and grew within cellular elements of Cebpe $e^{-/-}$blood (Figure 1I, left). In murine infection, H\&E staining of infected skin showed Cebpe $e^{-/-}$neutrophils full of intracellular S. aureus. By contrast, only a few intracellular bacteria were found at the lesion site of WT mice (Figure 1I, right). Taken together, these data suggest that neutrophils from Cebpe $e^{-/-}$mice do not provide an antimicrobial benefit to the host.

NAM augments killing of S. aureus by a C/EBPE-dependent mechanism. Because C/EBPe plays a critical role in the host immune response against $S$. aureus infection, we hypothesized that increased expression of C/EBPE could enhance immune killing of bacteria. As proof of principal, we induced overexpression of C/EBPE in a differentiated myeloid cell line $(45,46)$. U937 cells were stably transfected with a zinc-inducible C/EBPE expression vector (pMTE) and differentiated using phorbol 12-myristate 13-acetate (PMA). Interestingly, the differentiated U937 cells with forced expression of C/EBPE killed up to 30-fold more $S$. aureus compared with vector control (Figure 2). Zinc alone had no effect on the viability and growth of $S$. aureus (data not shown). 

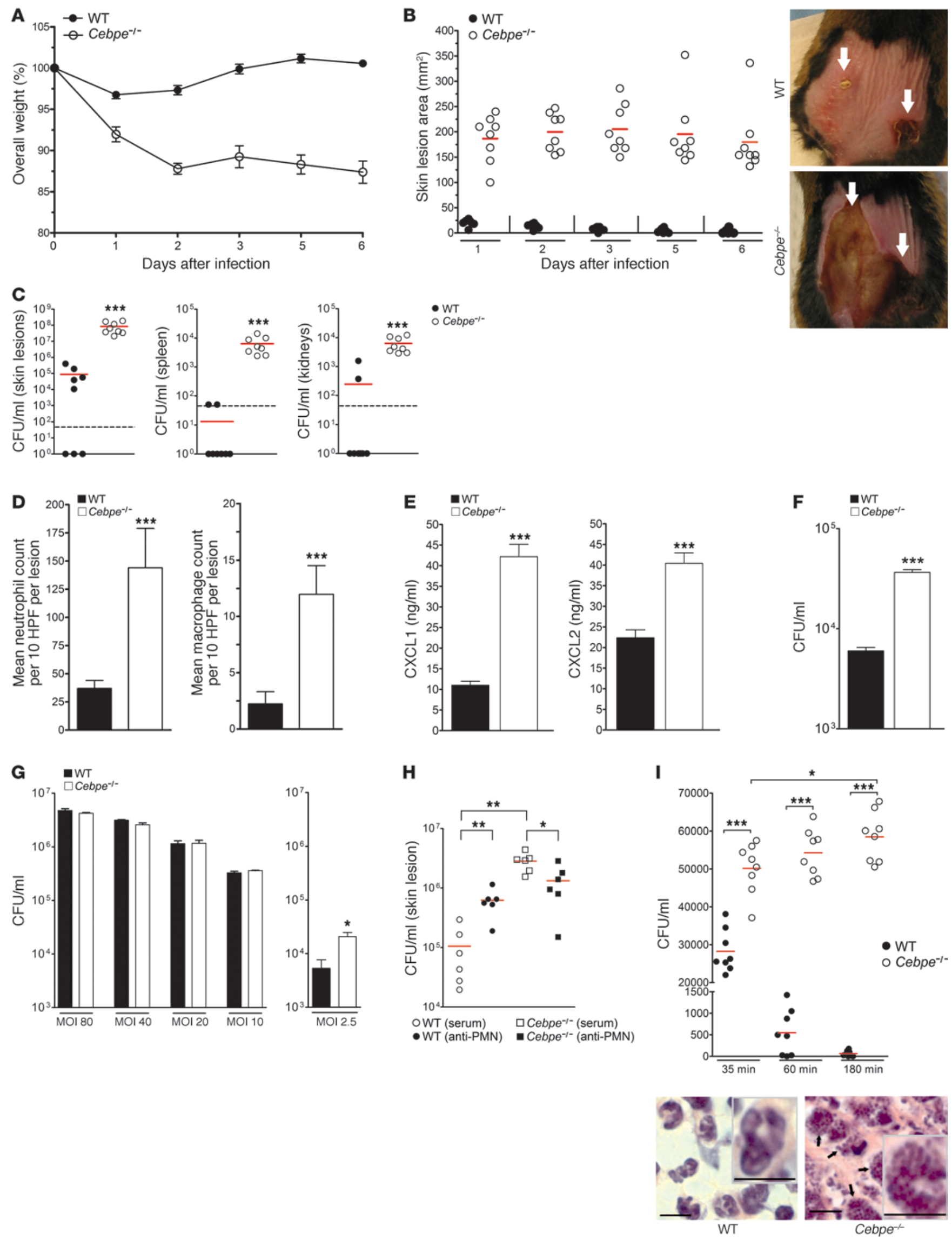


\section{Figure 1}

Impaired response of Cebpe ${ }^{-/-}$mice to $S$. aureus infection. (A-E) WT and Cebpe ${ }^{-/}$mice ( $n=8 /$ group) were injected s.c. with S. aureus (left flank, $\sim 2 \times 10^{7} \mathrm{CFUs}$; right flank, $\sim 1 \times 10^{8} \mathrm{CFUs}$ ). (A) Overall body weight as percentage of original weight (mean \pm SEM). $P<0.001$. (B) Area of skin lesions $(P<0.001)$. Representative images of skin lesions on day 6 p.i. Arrows point to lesions from $\sim 2 \times 10^{7}$ (left) and $\sim 1 \times 10^{8}$ (right) inocula. (C) CFUs from skin lesions, spleens, and kidneys on day 6 p.i. Dashed lines indicate limit of detection. (D) Neutrophil and macrophage counts from H\&E-stained skin lesions 24 hours p.i. (E) CXCL1 and CXCL2 measured from skin lesions at 24 hours p.i. $(n=10$ lesions/group). (F) Survival of $\sim 1.2 \times 10^{4} \mathrm{CFU} / \mathrm{ml}$ S. aureus in peripheral blood from WT and Cebpe ${ }^{-/}$mice ( $n=5 /$ group). (G) Bacterial clearance by BMDMs from WT and Cebpe $e^{-/-}$mice $(n=3 /$ group). Data in D-G are mean \pm SEM. (H) WT and Cebpe ${ }^{-/}$mice ( $n=6 /$ group) were treated with anti-PMN antibodies or normal serum daily for 4 days and infected s.c. on day 2 with $\sim 4 \times 10^{6} \mathrm{CFU}$ S. aureus. CFUs recovered from skin lesions on day 4 p.i. (I) Intracellular survival assay using whole blood from WT and Cebpe ${ }^{-/-}$mice ( $n=8$ /group). Representative histology (H\&E) of infected skin lesions from WT and Cebpe ${ }^{-/}$mice showing neutrophils with intracellular $S$. aureus (arrows). Scale bar: $10 \mu \mathrm{M}$. Throughout, symbols indicate individual samples, and red bars indicate mean. ${ }^{\star} P<0.05 ;{ }^{* \star} P<0.01 ;{ }^{* *} P<0.001$.

Based on the above findings, we next asked whether a pharmacologic agent could induce overexpression of C/EBPE in myeloid cells and thereby promote more effective immune-mediated killing of S. aureus. Skokowa and colleagues recently demonstrated that NAM could modify and enhance expression of C/EBP $\alpha$ and $\mathrm{C} / \mathrm{EBP} \beta$ (42). Therefore, in this study, we tested whether NAM, as a well-established HDAC inhibitor, could also modulate the activity of C/EBPe.

Peripheral blood drawn from 12 healthy human volunteers was pretreated ex vivo with either NAM ( $1 \mathrm{mM}$ or $10 \mathrm{mM})$ or PBS for 24 hours prior to infection with different inocula of S. aureus. NAM treatment of human neutrophils resulted in elevated protein levels of C/EBPE (Figure 3A, left). Associated with this change, NAM treatment reduced the survival of the pathogen in whole blood by 100 to 1,000 fold at 3 hours p.i. compared with that after PBS treatment (Figure 3A, right, and Supplemental Figure 5).

Notably, in our experiments, we used a NAM concentration of $1 \mathrm{mM}$, which is similar to the plasma concentration previously measured in humans treated with NAM (47). Importantly, NAM had no direct antistaphylococcal activity when incubated in the absence of phagocytic cells (Figure 3B and Supplemental Figure 6 ). As a further control, the NAM used in our study has been cell culture tested and was confirmed to be free of endotoxin (pyrogen) (Supplemental Figure 7).

To verify that the ex vivo effect of NAM is not related to altered viability of myeloid cells, we treated human blood with either NAM or PBS ex vivo and evaluated the survival of myeloid cell populations after 24 hours by complete blood count (CBC) and trypan blue staining. Consistently, we showed no significant decrease in neutrophil viability after 24 hours of treatment with either PBS or NAM (Figure 3C and Supplemental Figures 8 and 9). Likewise at 24 hours, ex vivo NAM treatment had no significant impact on the viability of mononuclear cells compared with that of PBS treatment (Figure 3C and Supplemental Figure 8).

Next, to determine the C/EBPE dependency of $S$. aureus killing, we isolated peripheral blood from WT and Cebpe - $^{-/}$mice, pretreated the blood for 24 hours with either NAM (1 mM) or PBS (control), and then infected the blood with different inocula of S. aureus. Consistent with findings in human blood, in the WT groups, NAM pretreatment enhanced killing of $S$. aureus by more than 1,000 fold compared with that in PBS controls (Figure 3D and Supplemental Figure 10). By contrast, in Cebpe $e^{-/}$groups, NAM pretreatment had no impact on the number of surviving S. aureus CFUs compared with that in PBS control groups (Figure 3D and Supplemental Figure 10). Pretreatment of WT murine peripheral blood with NAM for only 4 hours (instead of 24 hours) did not result in CFU differences (Supplemental Figure 11). Notably, the shown difference in the clearance of S. aureus by WT and Cebpe $e^{-/-}$blood was more modest in Figure 3D compared with that shown in Figure 1I. This could be attributed to differences in assay condition (standard whole blood assay versus gentamicin protection assay in whole blood): since C/EBPE-related granule proteins play a critical role in the killing of internalized bacteria, adjunctive killing of nonphagocytosed S. aureus by antibiotics, as shown in Figure 1I, could have accentuated the difference between WT and Cebpe $e^{-/-}$blood cell killing.

To test the critical role of C/EBPE in NAM-augmented killing in vivo, we injected WT and Cebpe $e^{-/-}$mice daily with either NAM ( $250 \mathrm{mg} / \mathrm{kg}$ ) or PBS i.p., starting 24 hours prior to systemic (i.p.) infection with $S$. aureus. This dose has routinely and safely been used in other studies $(48,49)$. Strikingly, after 48 hours, WT mice treated with NAM showed approximately 100 -fold lower S. aureus CFUs in the spleens and kidneys compared with that in PBS controls (Figure 3E). In contrast to our findings in WT mice, NAM treatment had no impact on the number of bacterial CFUs recovered from the spleens and kidneys of Cebpe $e^{-/}$mice at 48 hours p.i. (Figure 3E). NAM treatment was also investigated against a strain of MRSA and enhanced clearance of the MRSA compared with PBS treatment (Supplemental Figure 12). These findings are consistent with the hypothesis that C/EBPE is important in the immunomodulatory activity of NAM.

NAM selectively enhances neutrophil killing of S. aureus. To determine the specific contribution of the myeloid cell lineages to $S$. aureus clearance in whole blood, we isolated neutrophil and mononuclear fractions from the blood of healthy human volunteers. We pretreated the cells with either PBS or NAM (1 mM) for 24 hours and then measured intracellular survival of $S$. aureus in each myeloid cell population using a standard gentamicin protection assay. We showed that NAM augmented killing of $S$. aureus by neutrophils (Figure 4A and Supplemental Figure 13) but not by monocytes (Figure 4B and Supplemental Figure 14). We also evaluated the impact of NAM on macrophage clearance of $S$. aureus. WT murine BMDMs showed improved killing of $S$. aureus after NAM treatment (by less than 2 fold; Supplemental Figure 15), but the effect was much less dramatic than that observed with neutrophils.

Our ex vivo assays suggest that neutrophils are an important target of NAM antimicrobial activity. To investigate the critical role of neutrophils in vivo, we administered control or anti-PMN antibody to WT mice, as previously described above, and then measured S. aureus clearance either with or without NAM treatment. We showed that, in the absence of neutrophils, NAM had no effect on the clearance of $S$. aureus (Figure 4C). It is notable that, in the absence of neutrophils, mice exhibited a higher $S$. aureus burden that could potentially impact the effect of NAM on bacterial clearance. We repeated the experiment with a 10- to 20 -fold lower inoculum and showed that NAM had no effect with the reduced bacterial load (Supplemental Figure 16). 


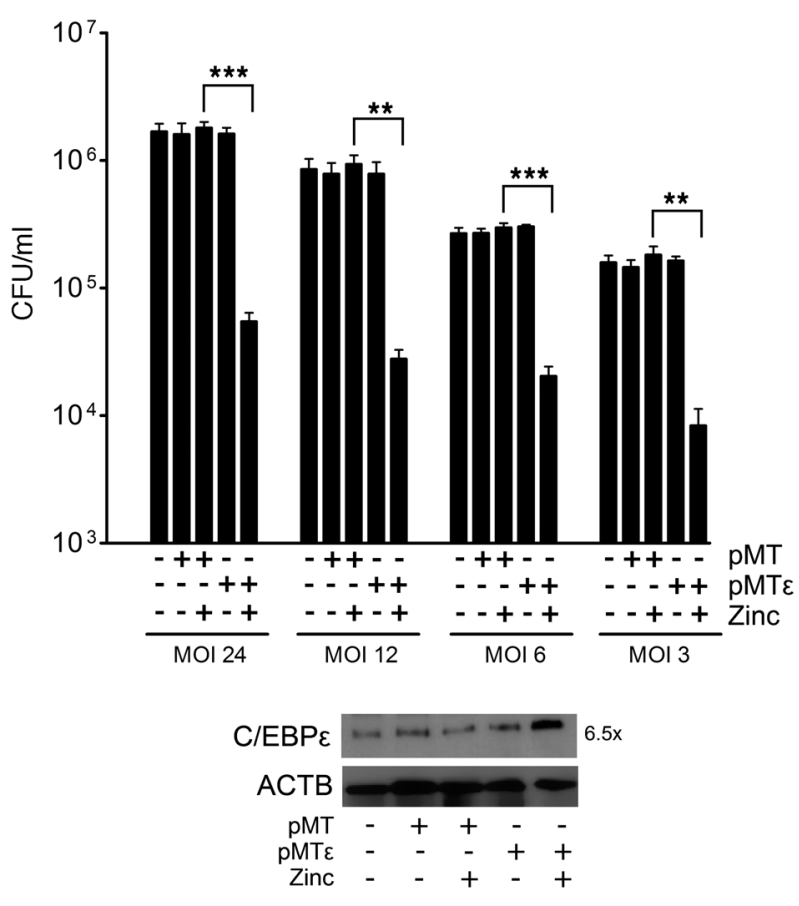

A previous report showed that administration of NAM to human volunteers can induce $\mathrm{C} / \mathrm{EBP} \alpha$ and $\mathrm{C} / \mathrm{EBP} \beta$ expression and granulocytosis (42). To determine whether an increased number of circulating neutrophils explains, at least in part, the antimicrobial activity of NAM in our in vivo infection model, we administered either NAM $(250 \mathrm{mg} / \mathrm{kg})$ or PBS daily to mice and monitored their changes in myeloid cell populations over 3 days. We showed that under our study conditions, NAM treatment did not induce an increase in myeloid cell populations after 1, 2, or 3 days (Figure 4D and Supplemental Figure 17).

$N A M$ induces expression of antimicrobial effectors downstream of $C / E B P \varepsilon$. Because NAM killing of $S$. aureus appears to be C/EBPE dependent, we next investigated whether NAM activates the expression of antimicrobial effectors known to be downstream of C/EBPE.

Upon exposing human neutrophils to NAM ( $1 \mathrm{mM})$ for 6 to 12 hours, we detected a 5 -fold increase in the level of acetylation on core histone $\mathrm{H} 3$ at the promoter region of CEBPE (Figure $5 \mathrm{~A}$ ) and 4-fold increase in acetylation of C/EBPE (Figure 5B and Supplemental Figure 18). This was accompanied by elevated protein levels of C/EBPE and downstream antimicrobial targets CAMP and LTF (Figure 5C and Supplemental Figures 19 and 20). Treatment of WT murine neutrophils also induced protein expression of C/EBPE and LTF but not of CAMP (Figure 5D). By contrast, NAM treatment of Cebpe $e^{-/-}$murine neutrophils had no effect on the level of CAMP or LTF (Figure 5D), suggesting that NAM induction of LTF occurs through the activation of C/EBPE. We also probed for gp91phox (NOX2) expression and reactive oxygen species production in neutrophils and found that NAM had no impact (Supplemental Figure 21).

We also investigated the effect of NAM on other myeloid cell lineages. We showed that NAM had no impact on C/EBPE, CAMP, and LTF expression in human monocytes (Figure 5E) but caused somewhat increased expression of C/EBPE and antimicrobial targets in macrophages (Supplemental Figures 22-24). The increased transcriptional activity of C/EBPe in macrophages was accompa-

\section{Figure 2}

Induced overexpression of C/EBP $\varepsilon$ promotes killing of $S$. aureus in vitro. The promonocytic cell line U937 was stably transfected with either PMTE or vector control (pMT), differentiated using PMA, and treated with or without zinc $(100 \mu \mathrm{M})$. The PMA-derived U937 cells were infected with S. aureus (Pig1) at multiple different MOI (bacte$\mathrm{ria} /$ macrophage) for 24 hours; data are mean \pm SEM. Western blot revealed a 6.5 -fold $(6.5 x)$ increase in $\mathrm{C} / \mathrm{EBP} \varepsilon$ protein expression in lysates from the infected zinc-treated cells carrying $\mathrm{PMT} \varepsilon$, compared with that of the controls.

nied by increased acetylation of histone $\mathrm{H} 3$ in the promoter region of CEBPE as well as increased protein acetylation (Supplemental Figures 22 and 23). In differentiated U937 myeloid cells stably transfected with $\mathrm{pMT}$, forced expression of C/EBPE directly promoted increased levels of LTF and CAMP (Figure 5F).

In addition, we administered NAM systemically to noninfected WT mice $(250 \mathrm{mg} / \mathrm{kg} / \mathrm{d}$, i.p.) and measured expression of Cebpe and downstream antimicrobial factors in murine bone marrow mononuclear cells. Expression analysis in these cells revealed increased levels of Cebpe, Camp, and Ltf mRNA and proteins after 72 hours of NAM treatment (Supplemental Figures 25-27).

Prior studies have documented a role of Th17 cells in the clearance of $S$. aureus (50). Therefore, we also evaluated whether T cells and murine protein of IL-17 and IFN- $\gamma$ are impacted by treatment with NAM. As shown in Supplemental Figure 28, splenocytes taken from mice administered either NAM or PBS daily for 3 days, with or without $S$. aureus infection, did not secrete different levels of murine protein of IL-17a and IFN- $\gamma$ following in vitro restimulations.

NAM augmented killing of S. aureus in vivo when given after the infectious challenge. To evaluate whether NAM is beneficial for treatment of an existing infection, we established systemic infection in WT mice with $S$. aureus for 12 hours prior to commencing daily treatment with NAM (Figure 6A). After 60 hours of infection, the number of bacteria recovered from the spleens and kidneys was 30- to 1,000-fold lower in NAM-treated mice compared with that in PBStreated controls. These data indicate that NAM can be effective against $S$. aureus whether the compound is administered before or after infection is established.

In line with our data on $S$. aureus, we also showed that NAM pretreatment of human peripheral blood improved the outcome of infection with other important human pathogens, such as K. pneumoniae and $P$. aeruginosa (Figure 6B).

Taken together, our findings strongly suggest that increased expression of C/EBPE, induced by the epigenetic modulator NAM, can efficiently enhance the clearance of $S$. aureus both in vitro and in vivo.

\section{Discussion}

Steady advances in molecular medicine and genetics have helped broaden our understanding of the underlying pathophysiology of leukocyte disorders and provided a clearer representation of how cells and other factors of the immune system interact. In this study, we substantiated the clinical finding that patients with $\mathrm{C} / \mathrm{EBP \varepsilon}$ deficiency are highly prone to $S$. aureus infection. After $S$. aureus challenge, C/EBPE-deficient mice exhibited dramatic skin pathology, were unable to clear $S$. aureus at the infection site, and had permitted systemic spread of the bacteria to spleens and kidneys. The underlying defects of C/EBPE-deficient neutrophils are many and include the absence of critical antimicrobial factors such 


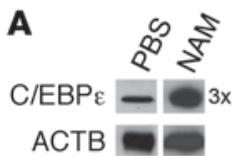

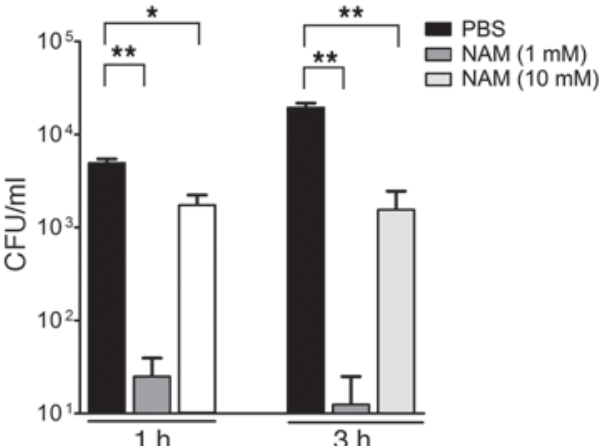

B

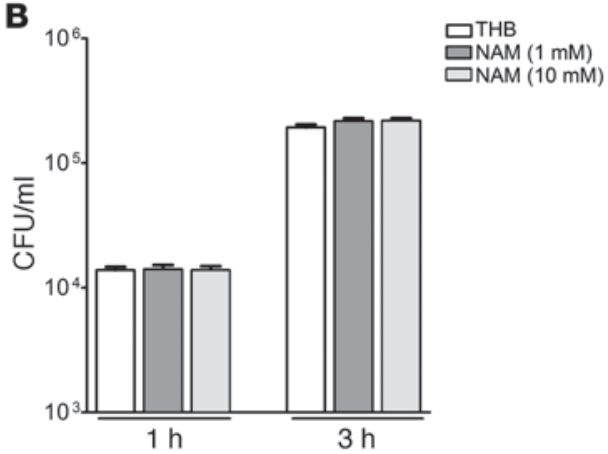

C $.0 \mathrm{~h}$

O PBS, $24 \mathrm{~h}$

$\square$ NAM $(1 \mathrm{mM}), 24 \mathrm{~h}$

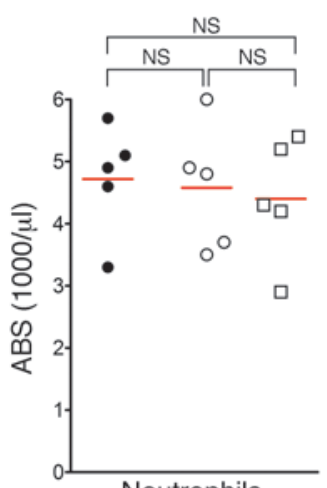

Neutrophils

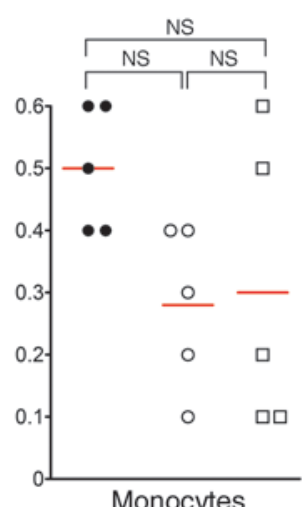

Monocytes
D

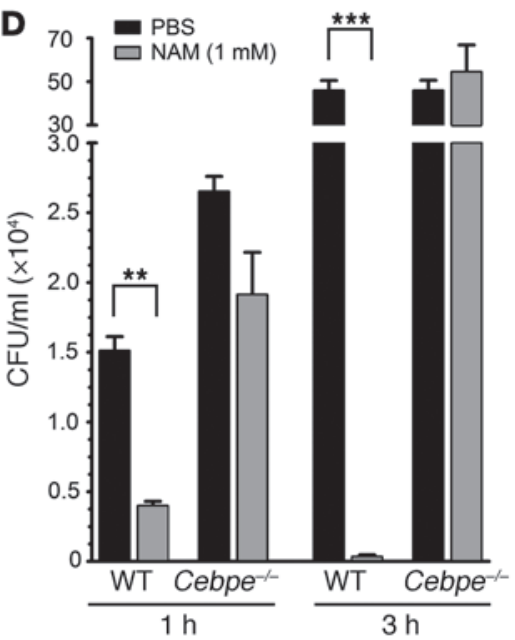

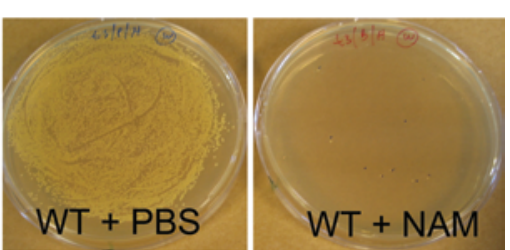

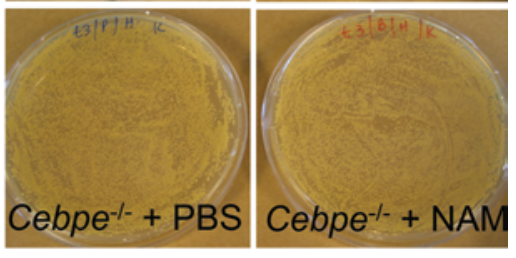

ONAM $(250 \mathrm{mg} / \mathrm{kg} / \mathrm{d})$

E
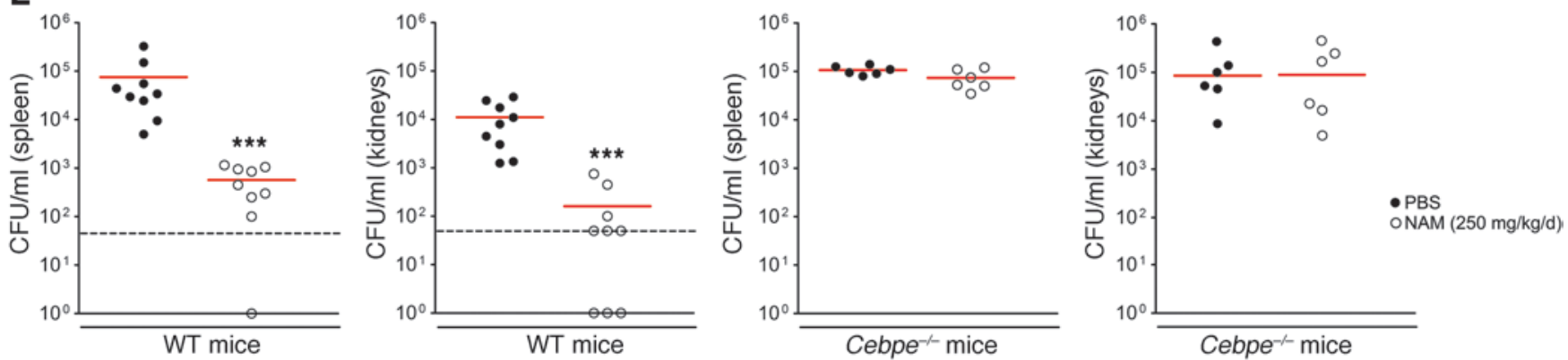

\section{Figure 3}

NAM augments killing of $S$. aureus by a C/EBPE-dependent mechanism. (A) Effect of NAM on clearance of $S$. aureus by human blood. Human blood $(n=4)$ was pretreated with NAM or PBS for 24 hours and inoculated with $S$. aureus $\left(6.5 \times 10^{3} \mathrm{CFU} / \mathrm{ml}\right)$. The number to the right of the blot indicates fold change compared with ACTB. (B) NAM does not have direct antistaphylococcal activity. S. aureus ( 104 CFU/ml) was treated with NAM or THB. (C) Effect of ex vivo NAM treatment on viability of human myeloid cells. Human blood $(n=5)$ was treated ex vivo with PBS or 1 mM NAM, and CBC was performed to determine the absolute (ABS) neutrophil and monocyte count for each volunteer at 0 and 24 hours. (D) Effect of NAM on clearance of $S$. aureus by murine blood. Blood from WT and Cebpe ${ }^{--}$mice $(n=6 /$ group $)$ was treated with NAM or PBS for 24 hours and then inoculated with S. aureus $\left(\sim 10^{4} \mathrm{CFU} / \mathrm{ml}\right)$. Images show CFUs after 3 hours. (E) Effect of NAM on in vivo clearance of $S$. aureus. WT $(n=9)$ or Cebpe ${ }^{-/-}$mice $(n=7)$ were treated daily with NAM or PBS, beginning 24 hours prior to i.p. S. aureus ( $\left.10^{7} \mathrm{CFUs}\right)$ infection. CFU counts in WT and Cebpe $\mathrm{e}^{-/-}$mice at 48 hours p.i. Dashed lines indicate limit of detection. One out of seven Cebpe ${ }^{-/-}$mice from each treatment group died and were excluded from analysis. (A, B, and D) Data are mean \pm SEM. (C and D) Red bars indicate mean. Symbols indicate individual samples. ${ }^{\star} P<0.05 ;{ }^{* \star} P<0.01 ;{ }^{* \star \star} P<0.001$.

as LTF and cathelicidin (e.g., CAMP), which are likely to contribute to the dramatic infection phenotype. In vivo, increased numbers of these phagocytic cells not only failed to compensate for the severe functional defects, but paradoxically appeared to contribute to the severity of infection, since depletion of the defective neutrophils improved clearance of S. aureus and reduced the size of necrotic lesions. We showed that ineffective clearance of S. aureus by Cebpe $e^{-/-}$ neutrophils permitted $S$. aureus to thrive within neutrophils and may further aggravate the infection. We, thereby, provide strong evidence that this transcription factor is essential for neutrophils, 

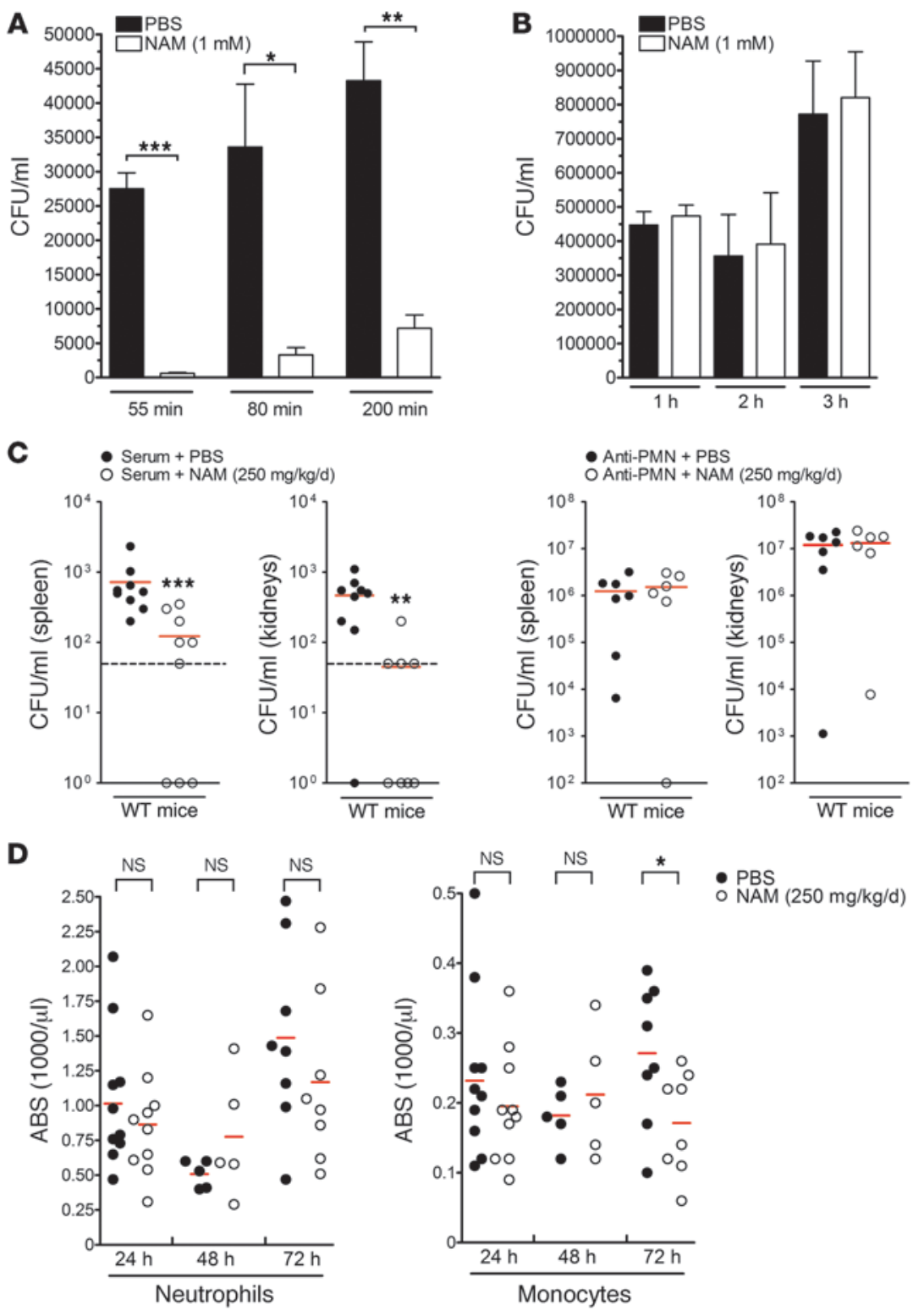

\section{Figure 4}

NAM selectively enhances neutrophil killing of S. aureus. (A) Effect of NAM on clearance of $S$. aureus by human neutrophils. Human neutrophils ( $n=3$ volunteers) were treated ex vivo with PBS or NAM (1 mM) for 20 hours and then infected with $2.4 \times 10^{8} \mathrm{CFU} / \mathrm{ml}$ preopsonized $S$. aureus in a gentamicin protection assay. Surviving intracellular bacteria recovered at indicated times (mean \pm SEM) are shown. (B) Effect of NAM on clearance of $S$. aureus by human peripheral mononuclear cells. Human monocytes ( $n=3$ volunteers) were treated ex vivo with PBS or NAM (1 mM) for 20 hours and then infected with preopsonized $S$. aureus. Recovered intracellular bacteria (mean \pm SEM) are shown. (C) Effect of NAM on in vivo clearance of $S$. aureus by WT mice depleted of neutrophils. WT mice ( $n=6-9 /$ group) were treated daily with NAM (250 mg/kg/d, i.p.) or with PBS beginning 24 hours prior to systemic (i.p.) infection with $\sim 1 \times 10^{7} \mathrm{CFU}$ $S$. aureus. Neutrophil depletion (or serum control) was performed in parallel. CFU count in spleens and kidneys of WT mice at 48 hours p.i. Dashed lines indicate limit of detection. (D) Effect of NAM treatment on granulocytosis in WT mice. WT mice ( $n=5-12 /$ group/time point) were treated with NAM ( $250 \mathrm{mg} / \mathrm{kg} / \mathrm{d}$, i.p.) or PBS, and CBC with automated differential was performed on blood taken at 24,48 , and 72 hours to determine the population of neutrophils and monocytes. (C and $\mathbf{D})$ Red bars indicate mean. Symbols indicate individual samples. ${ }^{\star} P<0.05 ;{ }^{* *} P<0.01 ;{ }^{* * *} P<0.001$. not only to avoid intracellular bacterial growth, but also to actively clear $S$. aureus, ensuring an effective innate immune system.

The profound phenotype of infected C/EBPE-deficient mice suggests that this myeloid-specific factor controls a critical antimicrobial program tailored for killing of $S$. aureus. Preliminary data on $K$. pneumoniae and $P$. aeruginosa in human blood experiments suggest a similar mechanism playing a role in other pathogens as well. Low plasma levels of C/EBPE-regulated antimicrobials have been shown to be predictive of increased risk of death attributable to infection in humans (51). Based on the dramatic infection phenotype of the C/EBPE-deficient mice, we hypothesized and demonstrated that enhanced transcriptional activity of C/EBPE in normal subjects, either by induced overexpression or by induction with NAM, could have a significant and often dramatic effect on killing of $S$. aureus both in vitro and in vivo. The absence of improved bacterial clearance in our $\mathrm{C} / \mathrm{EBP} \varepsilon$-deficient models further underlines the interplay between C/EBPE and NAM.
HDAC inhibitors, such as NAM, influence transcriptional expression by controlling chromatin condensation and regulate proteins involved in acetylation (23-27). As a precursor to $\mathrm{NAD}^{+}$, NAM can block deacetylation and the regeneration of $\mathrm{NAD}^{+}$ through interception of an ADP-ribosyl-enzyme-acetyl peptide intermediate $(29-31,38,52)$. $\mathrm{NAD}^{+}$-dependent transcriptional regulation was previously demonstrated for the highly conserved family members, C/EBP $\alpha$ and C/EBP $\beta$ (42). Moreover, transcriptional activity mediated by $\mathrm{C} / \mathrm{EBP} \beta$ can be enhanced by increased acetylation of its lysine residues by the HDAC inhibitors NAM and trichostatin (28). In line with these findings, we suggest that $\mathrm{NAM}$, in its role as an epigenetic modulator, can increase the protein expression of a select number of downstream targets mediated by $\mathrm{C} / \mathrm{EBP} \varepsilon$, including the well-recognized antimicrobials CAMP and $\operatorname{LTF}(15,19,53)$. Among myeloid cell populations, neutrophils appear to be a particularly important target of NAM for the clearance of $S$. aureus. Notably, and in contrast to human neutrophils, 
A Human neutrophils - ChIP

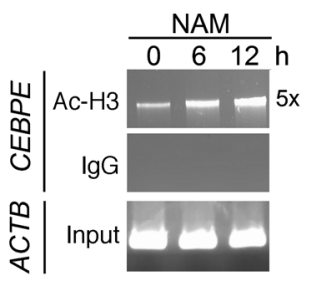

B

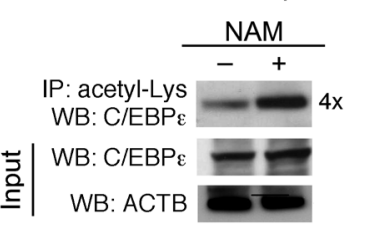

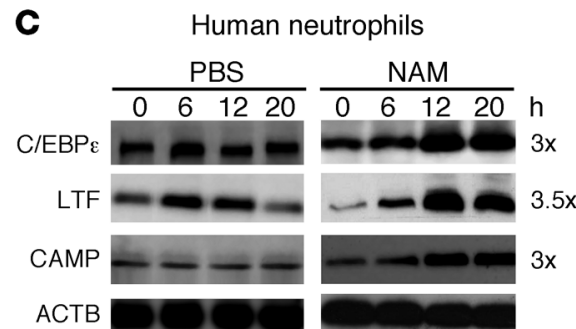

D

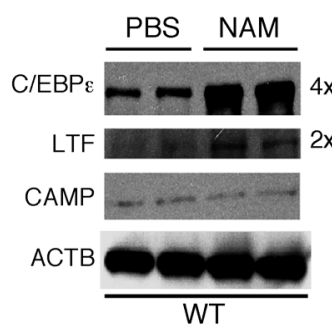

Murine neutrophils

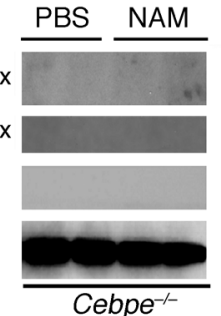

E

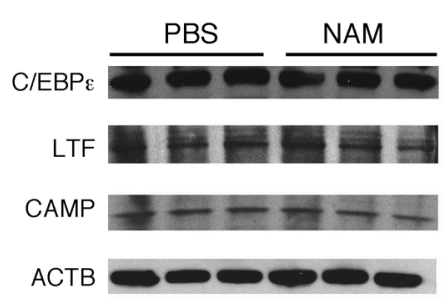

F

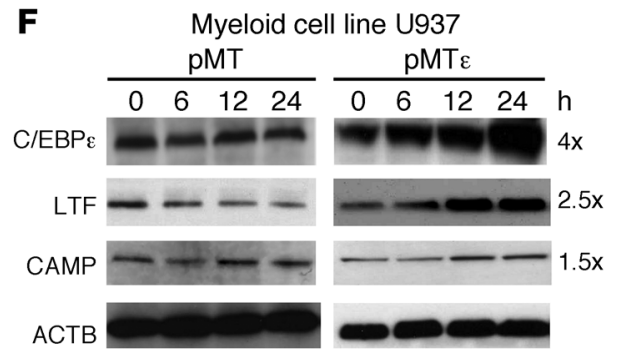

Figure 5

NAM induces expression of antimicrobial effectors downstream of C/EBP $\varepsilon$. (A) Effect of NAM on histone acetylation in human neutrophils. Blood was treated ex vivo with $1 \mathrm{mM}$ NAM. Neutrophils were isolated, and ChIP was performed using an antibody against acetylated histone H3 (Ac$\mathrm{H} 3$ ) or IgG control. The samples were analyzed by PCR using primers specific for the CEBPE promoter region. Input chromatin was included as a positive control using primers for the ACTB gene. (B) Effect of NAM on acetylation of C/EBP $\varepsilon$ protein in human neutrophils. Neutrophils isolated from blood were treated with $1 \mathrm{mM}$ NAM for 6 hours, and lysates were subjected to immunoprecipitation with an antibody against pan-acetylated lysine residues (acetyl-Lys), followed by Western blot (WB) with an antibody against C/EBPE. (C) Levels of C/EBPE, LTF, and CAMP in human neutrophils treated ex vivo with PBS or $1 \mathrm{mM} \mathrm{NAM}$. (D) Levels of C/EBPE, LTF, and CAMP in neutrophils isolated from WT and Cebpe-/- mice ( $n=15 /$ genotype) treated ex vivo with PBS or $1 \mathrm{mM}$ NAM for 20 hours. (E) Levels of C/EBPE, LTF, and CAMP in human monocytes treated ex vivo with PBS or $1 \mathrm{mM}$ NAM for 20 hours. (F) Forced expression of C/EBPE directly promoted increased levels of LTF and CAMP. (A-F) Fold changes indicated were determined by densitometry ( 24 hours versus 0 hours). ( $\mathbf{A}-\mathbf{C}$ and $\mathbf{E}$ ) Data represent 1 out of 3 human donors. For all instances, when human neutrophils or monocytes were used, blood from at least 3 human volunteers was used. Numbers to the right of blots indicate fold change compared with ACTB.

WT mouse neutrophils did not show increased CAMP expression when stimulated with NAM. This may reflect some difference between $\mathrm{C} / \mathrm{EBPE}$-mediated regulation of CAMP gene expression in humans and mice and is currently being investigated by our laboratories. We also probed for an additional antimicrobial target (NGAL) downstream of C/EBPE and found its expression was not affected by NAM in both human and mouse neutrophils (data not shown). Currently, we do not know which specific antimicrobial or antimicrobial combination downstream of C/EBPE is responsible for the clearance of $S$. aureus. However, since C/EBPE is known to be an essential regulator for the functional maturation of neutrophils, we speculate that C/EBPE may control a critical antimicrobial program that is inducible by NAM.

This program most likely controls specific elements of the neutrophil granules, which include a mixture of cytotoxic enzymes and peptides (e.g., LTF and CAMP), which bear not only a direct but also an indirect antibacterial effect by inducing the expression of cytokines and chemoattractants (54) as well as having a role in antigen presentation (55). It is notable that many neutrophil responses, including chemotaxis, exocytosis, respiratory burst activity, and chemokine synthesis, are mediated by p38MAPK, which is upstream and a direct activator of C/EBPE (56). Therefore, activation of upstream molecules regulating the activity of $\mathrm{C} / \mathrm{EBP} \varepsilon$ could be a further mechanism of how NAM exerts its antimicrobial effect. Dissecting the upstream and downstream components of the C/EBPE pathway will be an active area of future studies.
In our study of the myeloid cells, macrophages and monocytes, in comparison to neutrophils, appear to be more modestly impacted by C/EBPE deficiency or by NAM treatment for killing of S. aureus. Prior studies, however, have shown that C/EBPE-deficient macrophages have reduced expression of a number of immunerelated genes (e.g., Il18, Il10, $m c p 3$, and Serpinb2) (16). We, therefore, speculate that C/EBPE expression in macrophages may still impact clearance of $S$. aureus indirectly through cytokine responses. However, neutrophils appear to have a critical role in C/EBPE-mediated responses to $S$. aureus. Future studies using hematopoietic reconstitution after myeloablative irradiation could directly address the central role of the transcription factor C/EBPE in hematopoietic cells as the critical mediator of $S$. aureus killing.

An important concern that arises from the study is whether the therapeutic effect documented with $S$. aureus could be achieved in human subjects using safe NAM doses. In human trials, NAM is frequently administered as a modifier to patients undergoing radiotherapy $(47,48,57,58)$. In these trials, a plasma concentration of $1 \mathrm{mM}$ NAM is routinely achieved, a concentration that we used in our peripheral whole blood killing assays to demonstrate NAM efficacy. Therefore, our data suggest that a NAM concentration safely achievable in humans could provide protection against $S$. aureus infection. The $250 \mathrm{mg} / \mathrm{kg} / \mathrm{d}$ dosing in mice is a regimen that has been used safely in many murine studies $(48,49)$. That dose has not been widely tested in human subjects and is above the routine megadose regimen used in clinical trials $(80 \mathrm{mg} / \mathrm{kg} / \mathrm{d})$. However, stud- 
A

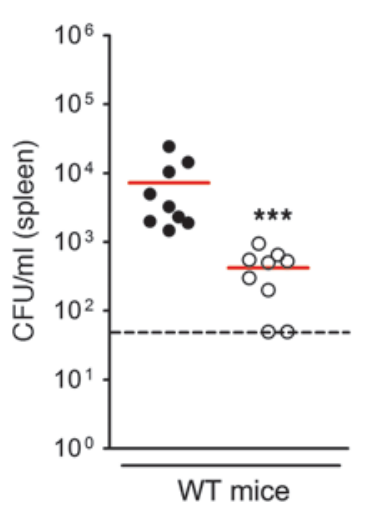

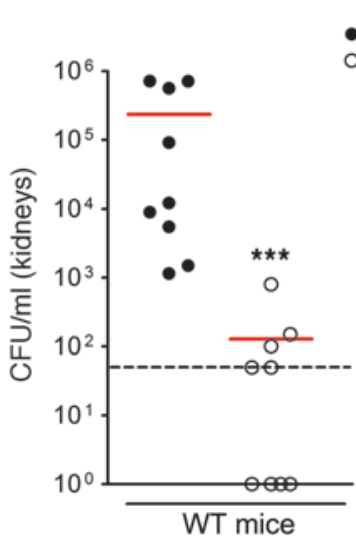

B

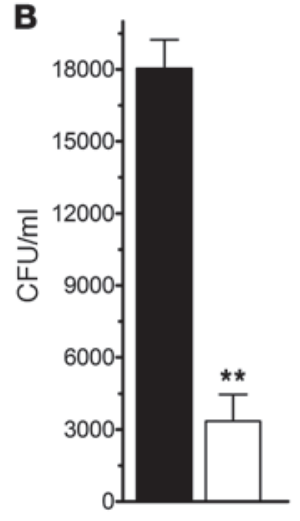

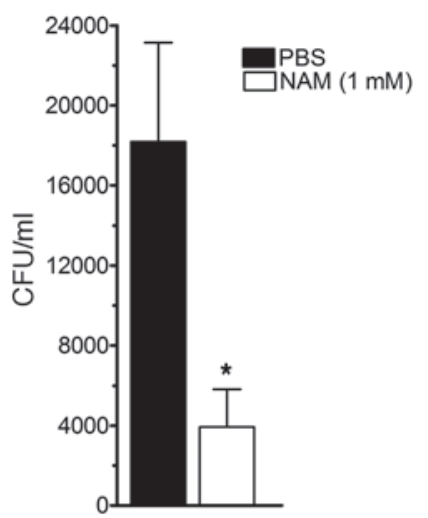

\section{Figure 6}

NAM augmented killing of $S$. aureus in vivo when given after the infectious challenge. (A) Effect of NAM in WT mice already infected with S. aureus. Animals ( $n=9 /$ group) were systemically infected i.p. with $\sim 2.0 \times 10^{7} \mathrm{CFU}$ S. aureus and treated daily with either NAM (250 mg/kg/d, i.p.) or with PBS (control), beginning 12 hours p.i. The CFU count in spleens and kidneys of WT mice at 60 hours p.i. is shown. Dashed lines indicate limit of detection. Red bars indicate mean. Symbols indicate individual samples. (B) NAM enhances clearance of $K$. pneumoniae and $P$. aeruginosa from the blood of healthy human volunteers. Bacterial counts (mean \pm SEM) recovered from the peripheral blood of human volunteers $(n=5)$ after the inoculation with $1.2 \times 10^{4} \mathrm{CFU} / \mathrm{ml} P$. aeruginosa (left) or K. pneumoniae (right). ${ }^{*} P<0.05 ;{ }^{* *} P<0.01 ;{ }^{* * *} P<0.001$.

ies have noted that the pharmacokinetics of NAM in humans and mice are quite different. In one report, an equivalent peak serum level was achieved with a dose of 80 to $90 \mathrm{mg} / \mathrm{kg}$ in humans and $171 \mathrm{mg} / \mathrm{kg}$ in mice (48). Additionally, a review of NAM safety concluded that though insufficient data are available to assess the upper level of safety in humans, with the newer preparations of NAM that are devoid of hepatotoxic contaminants, this is likely to be about $350 \mathrm{mg} / \mathrm{kg} / \mathrm{d}$ (59). Future studies will need to address the minimal safe NAM dose that induces clearance of $S$. aureus.

Also of clinical relevance, a previous study by Skokowa and colleagues showed that NAM treatment in humans at a dose of 10 to $20 \mathrm{mg} / \mathrm{kg} / \mathrm{d}$ induced neutrophilia in the blood of the patients at days 3 and 7 (42). The $250 \mathrm{mg} / \mathrm{kg} / \mathrm{d}$ dosing in mice that achieved enhanced clearance of $S$. aureus did not induce additional clearance at days 1 to 3 . However, given the recruitment of neutrophils with NAM administration in humans and the occasional association of excess neutrophils with immunopathology, human subjects receiving NAM for $S$. aureus infection would need to be monitored carefully for this side effect.

Our finding that NAM has an important effect on immunemediated killing of $S$. aureus in mouse and in human blood has a number of additional therapeutic implications. In an age when the number of antibiotics in the pipeline is limited and development of resistance occurs rapidly, use of complementary strategies to antibiotic treatment would provide a method of limiting development of antibiotic resistance. Because C/EBPE is a transcriptional activator of a number of important antimicrobial factors, induction of resistance to multiple host factors is less likely. Likewise, the use of an immune boosting strategy coupled with conventional antibiotics could provide important synergy.

While the use of NAM against conventional bacteria has not been previously reported, the vitamin compound has shown promising efficacy in treatment of $M$. tuberculosis infection, based on human studies performed in the 1960s (43). Similarly, NAM administration is associated with a beneficial effect in patients with HIV infection (43). Immune response to HIV is hypothesized to cause vitamin B3 deficiency, and the benefit of NAM supplementation comes from correction of this deficiency. In both cases, the role of C/EBPE in combating HIV and M. tuberculosis is unknown. Recently, Wurtele et al. reported that modulation of the yeast histone H3 Lys56 acetylation by NAM sensitized C. albicans to genotoxic and antifungal agents (44). We have demonstrated that NAM, as an HDAC inhibitor, can improve host defense and thereby promote bacterial clearance. NAM is not only effective against $S$. aureus, it also has demonstrated efficacy against other major human pathogens, such as K. pneumoniae and P. aeruginosa, in our human peripheral blood killing assay. These findings reflect the broad potential of compounds with the ability to stimulate the activity of C/EBPE and related antimicrobial targets. The effect of NAM and the associated contribution of C/EBPE and other downstream mediators in the treatment of pathogens are currently being explored by our laboratories.

In summary, we have demonstrated that $\mathrm{C} / \mathrm{EBP} \varepsilon$ is a regulatory factor that critically impacts the host's ability to fight $S$. aureus infections. Manipulation of C/EBPE expression in neutrophils and monocytes/macrophages, either by forced overexpression or by pharmacologic application of NAM, leads to a clear therapeutic effect on $S$. aureus infection. Our results constitute a proof of principle that compounds exerting modulatory effects of the myeloidspecific transcription factor C/EBPE may be suitable candidates for antimicrobial therapeutics.

\section{Methods}

Mice. Cebpe $e^{-/}$mice (12) and WT littermates were bred and maintained under pathogen-free conditions at an American Association for the Accreditation of Laboratory Animal Care-accredited animal facility at the Burns and Allen Research Institute at Cedars-Sinai Medical Center. Animals were housed in accordance with the procedures outlined in the Guide for the Care and Use of Laboratory Animals under an animal study proposal approved by the Cedars-Sinai Medical Center Institutional Animal Care and Use Committee. $\mathrm{Cebpe}^{-/-}$mice kept under the above mentioned conditions showed no signs of infection during life or at autopsy, with survivals equivalent to those of WT mice. Sex-matched mice used throughout our study were 6 to 10 weeks old. 
Bacterial strains and growth conditions. Unless otherwise indicated, WT S. aureus Pig1 (4), isolated from the skin of a child with atopic dermatitis, was used in the experiments. The MRSA clinical isolate COL (NRS100; NARSA) was also used.

S. aureus were propagated in Todd-Hewitt broth (THB; Difco) at $37^{\circ} \mathrm{C}$, with shaking at $250 \mathrm{rpm}$, or on THB agar (THA). Overnight bacterial culture was diluted until an optical density at $600 \mathrm{~nm}$, corresponding to approximately $10^{8} \mathrm{CFU} / \mathrm{ml}$, was reached. Bacteria were harvested by centrifugation at $3,300 \mathrm{~g}$ for 10 minutes at $4^{\circ} \mathrm{C}$ and then washed twice with $\mathrm{PBS}$ (without $\mathrm{Ca}^{2+}$ and $\mathrm{Mg}^{2+} ;$ Mediatech). S. aureus strains were routinely cultured on Tryptic Soy sheep blood agar plates, and colonies with comparable hemolysis phenotypes were selected for each experiment.

Murine skin infection model. S. aureus was pelleted, washed twice, and resuspended in PBS mixed 1:1 with sterile Cytodex beads (GE Healthcare), following an established protocol for generating localized $S$. aureus and S. pyogenes s.c. infection $(4,60)$. One hundred microliters of 2 separate inocula, as specified (see the legend for Figure 1), were administered by s.c. injection into the respective 2 flanks of each mouse. Injections were performed with careful visualization of the needle to assure that they were not intramuscular. Serial dilutions were prepared and plated to confirm the actual inocula used.

Determination of lesion size and tissue bacterial CFUs. Baseline weights of mice were recorded prior to infection and daily thereafter until sacrifice. Lesions were measured with a caliper, daily throughout infection. Lesions were defined by darkened areas of dermonecrosis. Our method to measure lesion size has been previously reported (61). Briefly, skin lesions were quantified by multiplying the length and width of the lesion. Irregularly shaped lesions were broken down into smaller symmetrical pieces, and each piece was measured by the same method.

Following euthanization of mice, infected skin lesion tissue was aseptically excised, thoroughly homogenized, and mixed in $1 \mathrm{ml}$ PBS, as previously shown (61). Ten-fold serial dilutions of the homogenates were plated on THA for CFU determination. The spleen and both kidneys were aseptically removed from each animal and processed in the same way. When required, the appropriate homogenized suspensions (skin lesions) were centrifuged at $15,000 \mathrm{~g}$ for 10 minutes, and supernatants were stored at $-80^{\circ} \mathrm{C}$ for subsequent analysis by ELISA.

Quantification of neutrophils and macrophages in infected skin lesions. WT and Cebpe $e^{-/}$mice ( $n=8$ per group) were infected by s.c. injection of S. aureus at the specified inoculum (see the legend for Figure 1) and sacrificed at 24 hours p.i. Infected tissues (skin lesions) were then excised and fixed in $10 \%$ formalin (Medical Chemical Corporation) overnight. Paraffin embedding and $\mathrm{H} \& \mathrm{E}$ staining were performed by the Department of Pathology at CedarsSinai Medical Center. Image acquisition was performed with the Zeiss Axio Imager M1 microscope and the AxioVision 4.6 software (Zeiss). A minimum of 3 mice (2 lesions per mouse) from each genotype were randomly selected for enumeration of macrophages and neutrophils. Two blinded observers counted the cells from 10 nonoverlapping high-power fields (original magnification, $\times 400$ ) within each lesion. The average count was obtained from each mouse in each group, and SEM was calculated from these averages.

ELISA. Mouse CXCL1-specific and CXCL2-specific ELISAs were performed according to the manufacturer's instructions (R\&D Systems).

Isolation of murine bone marrow mononuclear cells and cultivation of $B M D M s$. Bone marrow cells were harvested from WT or Cebpe $e^{-/-}$mice. Bone marrow was flushed out of isolated femurs and tibiae with RPMI 1640 medium and $10 \%$ heat-inactivated FBS using a 25 -gauge needle. Cells were then incubated for 4 hours in a humidified atmosphere at $37^{\circ} \mathrm{C}$ and $5 \% \mathrm{CO}_{2}$ to deplete adherent cells. Nonadherent bone marrow cells were cultured with $10 \mathrm{ng} / \mathrm{ml}$ murine M-CSF (Peprotech) in RPMI 1640 with $10 \%$ FBS for 7 days to induce BMDMs. Bone marrow mono- nuclear cells were isolated from nondepleted bone marrow cells using Lymphocyte Separation Medium (Mediatech).

Intracellular survival assay (gentamicin protection assay) in macrophages. Macrophages were seeded at a density of $5 \times 10^{4}$ cells per well $(100 \mu l)$ in 96-well tissue culture plates. Macrophages were infected with S. aureus at the specified MOI (see Figure $1 \mathrm{G}$ and Figure 2). To promote infection, bacteria were spun down onto the macrophages at $500 \mathrm{~g}$ for 10 minutes at room temperature, before incubating the cells in a humidified atmosphere at $37^{\circ} \mathrm{C}$ and $5 \% \mathrm{CO}_{2}$. After 30 minutes, macrophages were washed 3 times with prewarmed media to remove extracellular bacteria. Gentamicin (Invitrogen) was then added to each well at a final concentration of $400 \mu \mathrm{g} / \mathrm{ml}$ for 1.5 hours. At this time, the concentration of gentamicin in the media was reduced to $100 \mu \mathrm{g} / \mathrm{ml}$ for the remainder of the assay. At 24 hours p.i., cells were washed 3 times with PBS, then $100 \mu$ l of $0.02 \%$ Triton X-100 in water was added to each well and pipetted vigorously 10 times to promote macrophage lysis and release intracellular bacteria. Ten-fold serial dilutions of each cell lysate were immediately plated onto THA, and CFUs were enumerated following overnight incubation at $37^{\circ} \mathrm{C}$. Data are representative of at least 2 independent experiments performed in triplicate.

For assays involving PMA-differentiated human U937 macrophages, 24 hours prior to infection, media was replaced without G418 and PMA for the remainder of the time. $\mathrm{Zinc}\left(100 \mu \mathrm{M} \mathrm{Zn}_{2} \mathrm{SO}_{4}\right)$ was added to the respective $\mathrm{pMT \varepsilon}$ and control groups 24 hours prior to infection and was present for the remainder of the assay.

Neutrophil depletion in vivo. Depletion of neutrophils was carried out as described previously $(62,63)$. Briefly, mice were made neutropenic by i.p. administration of $150 \mu \mathrm{l}$ rabbit anti-mouse PMN antibody (Cedarlane Labs) 24 hours prior (day -1$)$ to s.c. infection with $S$. aureus on day 0 and every 24 hours thereafter, until sacrifice on day 4 . The manufacturer certified that the antibody was sterile and suitable for use in cytotoxic assays and in vivo depletion. Control groups received equal amounts of normal rabbit serum (Sigma-Aldrich; sterile-filtered, cell culture and endotoxin tested) by i.p. injection.

WT and Cebpe $e^{-/-}$mice receiving either anti-mouse PMN antibody or normal serum (control) were infected s.c. with S. aureus on day 0 (refer to Murine skin infection model). Skin lesion areas were measured daily, and on day 4 (sacrifice) the CFUs in skin lesions, spleens, and kidneys were determined.

To confirm depletion of neutrophils after antibody injection, WT mice ( $n=3$ /group) were sacrificed at day 0 and day 4 of infection. Splenocytes were collected after sacrifice and homogenized, and cells were stained with PE-anti-Ly6G monoclonal antibody and PE.Cy5-anti-CD11b monoclonal antibody (eBiosciences). The population of neutrophils $\left(\mathrm{Ly}_{6} \mathrm{G}^{+}, \mathrm{CD} 11 \mathrm{~b}^{+}\right)$ were determined using a CyAnTM flow cytometer (Beckman Coulter), and the data were analyzed by Summit (Dako) software. The total population of neutrophils of mice treated with antibody was highly reduced on day $0(-72 \%)$ and day 4 (-96\%; data not shown) compared with that of mice treated with normal serum.

Intracellular survival assay (gentamicin protection assay) in murine whole blood. Bacteria were pelleted, washed twice, diluted to $1.5 \times 10^{7} \mathrm{CFU} / \mathrm{ml}$ in $50 \mu \mathrm{l}$ PBS (without $\mathrm{Ca}^{2+}$ and $\mathrm{Mg}^{2+}$ ), and immediately mixed with $150 \mu \mathrm{l}$ of freshly drawn murine (WT or Cebpe $e^{--} ; n=8$ /group) peripheral whole blood in sterile heparinized 2-ml round-bottom Eppendorf tubes. Notably, blood from WT and Cebpe $e^{-/-}$mice contained approximately the same number of neutrophils per microliter (Supplemental Figure 4). Reactions were incubated at $37^{\circ} \mathrm{C}$ on a rotary shaker.

After 15 minutes of infection, gentamicin (Invitrogen; working stock, $5 \mathrm{mg} / \mathrm{ml}$ in PBS) was added to each $200 \mu \mathrm{l}$ blood reaction at a final concentration of $400 \mu \mathrm{g} / \mathrm{ml}$ to kill extracellular bacteria. At 75 minutes p.i., the concentration of gentamicin in the blood was reduced to approximately $130 \mu \mathrm{g} / \mathrm{ml}$ for the remainder of the assay by adding $240 \mu \mathrm{l}$ PBS to each blood reaction. 
Forty-microliter (35 and 60 minutes p.i.) or $120-\mu \mathrm{l}$ (180 minutes p.i.) aliquots were taken from each blood reaction, diluted into $500 \mu \mathrm{l}$ PBS (in sterile 0.6- $\mathrm{ml}$ Eppendorf tubes), and centrifuged at 15,000 $\mathrm{g}$ for 3 minutes at room temperature. After carefully removing the supernatant, pure water $(200 \mu \mathrm{l})$ was added and pipetted vigorously 10 times to promote host cell lysis and release intracellular bacteria. Each $200-\mu \mathrm{l}$ cell lysate was immediately plated onto THA using the spread-plate technique (30-ml agar plates to further dilute out any remaining gentamicin), and CFUs were enumerated following overnight incubation at $37^{\circ} \mathrm{C}$.

Development of U937-pMTE cells. As reported previously by our group (64), pMTE was constructed by inserting a full length of CEBPE cDNA at the XhoI and HindIII sites of the $\mathrm{pMTCB}^{+}$vector (pMT; gift from F.J. Rauscher III, The Wistar Institute, Philadelphia, Pennsylvania, USA). We used the human promonocytic U937 cell line (ATCC) stably transfected with pEGFP plasmid (Clontech Laboratories) and either zinc-inducible pMTE or control vector pMT. Cells were maintained at between $2 \times 10^{5}$ cells $/ \mathrm{ml}$ and $1 \times 10^{6}$ cells $/ \mathrm{ml}$ in RPMI 1640 medium (Invitrogen) supplemented with $10 \%$ heat-inactivated FBS (Gemini Bio-Products), $2 \mathrm{mM}$ L-glutamine, and G418 (neomycin, $900 \mu \mathrm{g} / \mathrm{ml}$; Omega Scientific) for selection. Multiple polyclonal cultures (>98\% GFP positive) were screened for zinc-inducible C/EBPE protein overexpression by Western blot analysis.

PMA-differentiated human U937 myeloid cells. U937 cells alone or carrying either PMTE or vector control were seeded at an appropriate density in tissue culture plates. The cells were subsequently induced to differentiate by addition of $10 \mathrm{ng} / \mathrm{ml}$ PMA (Sigma-Aldrich) for 24 hours.

Human blood. Participants in our study included healthy humans with a negative history of infection, no antibiotic treatments, and no immuneboosting supplements in the 4 weeks prior to donating blood. Peripheral blood was collected from individuals in a fasting condition. Blood was routinely collected in the presence of either heparin or $\mathrm{K}_{2}$ EDTA and was used immediately for downstream applications.

NAM. NAM $\left(\mathrm{C}_{6} \mathrm{H}_{6} \mathrm{~N}_{2} \mathrm{O} ; \mathrm{FW} 122.13\right)$, tested for cell culture and insect cell culture, was purchased from Sigma-Aldrich. On each occasion prior to use, NAM was prepared fresh in sterile endotoxin-free PBS (without $\mathrm{Ca}^{2+}$ and $\mathrm{Mg}^{2+}$ ) and sterilized through a nonpyrogenic $0.22-\mu \mathrm{m}$ low-protein-binding filter (PALL Life Sciences). Each specific lot of NAM used in our study was confirmed to be endotoxin-free (pyrogen-free) using the end-point chromogenic Limulus amebocyte lysate endochrome method (refer to Supplemental Methods and Supplemental Figure 7 for more detail).

Assessing the effect of NAM on the growth and viability of S. aureus. We assessed whether NAM, at the concentrations used in our study, adversely affect the growth and viability of $S$. aureus. PBS was chosen as an inert nongrowth medium, and THB was chosen as a suitable growth medium for $S$. aureus. Seventy-five microliters of NAM ( $1 \mathrm{mM}$ and $10 \mathrm{mM}$ final concentrations; in either THB or PBS) or THB or PBS alone (respective controls) were placed in sterile 2-ml round-bottom Eppendorf tubes and then inoculated with $S$. aureus $\left(\sim 1 \times 10^{4} \mathrm{CFU} / \mathrm{ml}\right.$ in $25 \mu$ l of PBS or THB $)$ and immediately briefly vortexed. Triplicate reactions were incubated at $37^{\circ} \mathrm{C}$ for 1,3 , and 6 hours on a rotary shaker, at which time 10 -fold serial dilutions were plated on THA for enumeration of CFUs.

In a different assay, $S$. aureus $\left(\sim 1 \times 10^{8} \mathrm{CFU} / \mathrm{ml}\right.$ in THB $)$ was incubated either with or without $50 \mathrm{mM}$ NAM. Triplicate $1-\mathrm{ml}$ reactions were incubated at $37^{\circ} \mathrm{C}$ on a rotary shaker, and at various time points $(6,12$, and 24 hours), 10 -fold serial dilutions were plated on THA for enumeration of CFUs. These assays were performed on 3 independent occasions.

Murine and human whole blood assays. This well-described phagocytic survival assay has been previously reported (4). Bacteria were pelleted, washed twice, diluted to the specified inoculum (see the legends for Figures 1, 3, and 6) in $25 \mu \mathrm{lPBS}$ (without $\mathrm{Ca}^{2+}$ and $\mathrm{Mg}^{2+}$ ), and immediately mixed with $75 \mu \mathrm{l}$ of freshly drawn human or murine peripheral whole blood in sterile heparinized 2-ml round-bottom Eppendorf tubes. Reactions (performed in minimum in triplicate) were incubated at $37^{\circ} \mathrm{C}$ for 1 to 3 hours on a rotary shaker, at which time 10 -fold serial dilutions were plated on THA for enumeration of surviving CFUs.

When required, freshly drawn human or murine peripheral blood was pretreated with NAM ( $1 \mathrm{mM}$ or $10 \mathrm{mM}$ ) or PBS (without $\mathrm{Ca}^{2+}$ and $\mathrm{Mg}^{2+}$ ) prior to inoculation with bacteria. Pretreatment was performed in sterile, nontreated, low evaporation tissue culture plates (Becton Dickinson) for 24 hours in a humidified atmosphere ( $95 \%$ humidity) at $37^{\circ} \mathrm{C}$ and $5 \% \mathrm{CO}_{2}$, with gentle mixing on a nutator. On each occasion, blood was aseptically taken from mice via cardiac puncture using a 22-gauge needle to minimize lysis and maintain the integrity of the blood for the duration of the respective assay.

Murine systemic infection. Mice were systemically infected by i.p. injection of $\sim 1 \times 10^{7} \mathrm{CFU} / \mathrm{ml}$ to $2 \times 10^{7} \mathrm{CFU} / \mathrm{ml}$ S. aureus for 48 hours. Following euthanasia, the spleen and both kidneys were removed for $\mathrm{CFU}$ determination.

CBC of human and mouse blood specimens. $\mathrm{CBCs}$ were used to measure the viability of myeloid lineages in human and mouse blood. Blood specimens were collected in BD Microtainer tubes with $\mathrm{K}_{2}$ EDTA (Becton Dickinson). Specimens were analyzed for hematology testing within 3 hours of collection and were stored in racks or continuously mixed at room temperature during this time.

Human blood specimens were routinely analyzed by the Department of Pathology at Cedars-Sinai Medical Center. CBC with automated differential was performed using a Beckman Coulter LH 1500 Series Hematology Automation System.

Murine blood specimens were routinely analyzed by the Department of Comparative Medicine at Cedars-Sinai Medical Center. CBC with automated differential was performed using a Hemavet 950 FS Veterinary Hematology System (Drew Scientific Inc.). Automated functionality included wbc, rbc, $\mathrm{Hb}, \mathrm{HCT}, \mathrm{MCV}, \mathrm{MCH}, \mathrm{MCHC}$, platelets, and 5-part wbc differential, including neutrophils, lymphocytes, monocytes, eosinophils, and basophils.

Isolation of human neutrophils. Polymorphprep (Axis-Shield) was used for the isolation of neutrophils from human peripheral blood according to the manufacturer's instructions.

Human neutrophils were maintained ex vivo in RPMI 1640 supplemented with $10 \%$ heat-inactivated FBS and $2 \mathrm{mM}$ L-glutamine in a humidified atmosphere at $37^{\circ} \mathrm{C}$ and $5 \% \mathrm{CO}_{2}$. When indicated, immediately following isolation, human neutrophils were treated ex vivo with either NAM (1 $\mathrm{mM}$ ) or PBS for 20 to 24 hours and subsequently used for Western blot or specific functional assays.

For some experiments, freshly drawn human peripheral blood was pretreated with either NAM (1 or $10 \mathrm{mM}$ ) or PBS (without $\mathrm{Ca}^{2+}$ and $\mathrm{Mg}^{2+}$ ), prior to isolating the neutrophils using Polymorphprep. Pretreatment of the human blood was performed in sterile, nontreated, low evaporation tissue culture plates (Becton Dickinson) for 20 to 24 hours in a humidified atmosphere $\left(95 \%\right.$ humidity) at $37^{\circ} \mathrm{C}$ and $5 \% \mathrm{CO}_{2}$, with gentle mixing on a nutator.

Trypan blue exclusion, according to standard staining protocol, was used to measure the viability (over time) of neutrophils in culture or neutrophils isolated directly from blood.

Isolation of human monocytes. Human peripheral blood was collected in the presence of heparin and used immediately. Blood was layered over Lymphocyte Separation media (Fisher Scientific) according to manufacturer's instructions and centrifuged at $1,000 \mathrm{~g}$ for 25 minutes at room temperature without the break engaged. The cell layer was isolated and washed 3 times with RPMI 1640. Monocytes were isolated using Human Monocyte Isolation Kit II (Miltenyi Biotec) according to the manufacturer's instructions. Briefly, the Monocyte Isolation Kit II is an indirect magnetic labeling system for the isolation of untouched monocytes from human peripheral blood mononuclear cells. Human monocytes were isolated by depletion of 
nonmonocytes (negative selection). Nonmonocytes were indirectly magnetically labeled with a cocktail of biotin-conjugated monoclonal antibodies, as primary labeling reagent, and anti-biotin monoclonal antibodies conjugated to MicroBeads, as secondary labeling reagent.

Human monocytes were maintained ex vivo in RPMI 1640 supplemented with $10 \%$ FBS, $2 \mathrm{mM}$ L-glutamine, and $50 \mathrm{ng} / \mathrm{ml}$ recombinant human M-CSF (PeproTech) in a humidified atmosphere at $37^{\circ} \mathrm{C}$ and $5 \%$ $\mathrm{CO}_{2}$. Human monocytes were treated ex vivo with either NAM (1 mM) or PBS for approximately 20 hours and subsequently used for Western blot or functional assays.

Isolation of mouse neutrophils. Isolation of neutrophils from murine whole blood was performed according to a protocol established by Cedarlane Labs. Blood was aseptically collected from mice via cardiac puncture using a 22-gauge needle to minimize lysis and maintain the integrity of the blood cells. Blood was collected in the presence of heparin and used immediately. $\mathrm{rbc}$ were pelleted by centrifugation at $1400 \mathrm{~g}$ for 10 minutes at room temperature, without the brake engaged. The buffy coat (wbc layer) and the top layer of the rbc was harvested with a Pasteur pipette and transferred to a fresh tube. PBS (without $\mathrm{Ca}^{2+}$ and $\mathrm{Mg}^{2+}$ ) was added to wash and fully suspend the cells, and the mixture was centrifuged $(600 \mathrm{~g}, 10$ minutes, room temperature) to re-pellet the cells. rbc were then lysed using standard protocol. wbc were subsequently resuspended in $4 \mathrm{ml}$ of a serum-free medium (PBS without $\mathrm{Ca}^{2+}$ and $\mathrm{Mg}^{2+}$ ). $4 \mathrm{ml}$ diluted wbc were then carefully layered over $3 \mathrm{ml}$ Lympholyte-Mammal (Cedarlane Labs) at room temperature in a $15-\mathrm{ml}$ centrifuge tube. The gradient was centrifuged at $800 \mathrm{~g}$ for $25 \mathrm{~min}$ utes at room temperature, without the brake engaged. The resulting interfacial band consisted of lymphocytes, and the pellet consisted of PMNs. Once the PMNs were obtained, further washing was carried out to remove any rbc membranes ( $\mathrm{rbc}$ ghosts). The majority of the granulocytes were neutrophils (>70\%).

Murine neutrophils were maintained ex vivo in RPMI 1640 supplemented with $10 \% \mathrm{FBS}$ and $2 \mathrm{mM}$ L-glutamine and incubated in a humidified atmosphere at $37^{\circ} \mathrm{C}$ and $5 \% \mathrm{CO}_{2}$. Murine neutrophils were treated ex vivo with either NAM $(1 \mathrm{mM})$ or PBS for approximately 20 hours and subsequently used for Western blot.

Intracellular survival assay (gentamicin protection assay) in buman neutrophils. S. aureus were opsonized with $10 \%$ prewarmed pooled human plasma and incubated for 20 minutes at $37^{\circ} \mathrm{C}$ under slow rotation. The preopsonized bacteria were pelleted, washed twice, diluted to the specified inoculum (see the legend for Figure 4) in $50 \mu \mathrm{l}$ RPMI, and immediately mixed with $150 \mu \mathrm{l}$ human neutrophils $\left(6 \times 10^{5}\right.$ total neutrophils) in sterile 2 - $\mathrm{ml}$ roundbottom Eppendorf tubes. Before being used in this assay, the neutrophils had been pretreated ex vivo with either NAM $(1 \mathrm{mM})$ or PBS for 20 hours. Based on $\mathrm{CBC}$ data from at least 11 healthy human donors, a mean of 4,000 neutrophils per microliter of human blood was assumed when determining an appropriate MOI to use in this assay. Reactions were incubated at $37^{\circ} \mathrm{C}$ on a rotary shaker.

After 20 minutes of infection, gentamicin (Invitrogen; working stock, $5 \mathrm{mg} / \mathrm{ml}$ in RPMI) was added to each $200 \mu \mathrm{l}$ neutrophil reaction at a final concentration of $400 \mu \mathrm{g} / \mathrm{ml}$ to kill extracellular bacteria. At 80 minutes p.i., the concentration of gentamicin in the reaction was reduced to approximately $130 \mu \mathrm{g} / \mathrm{ml}$ for the remainder of the assay by adding $240 \mu \mathrm{l} \mathrm{RPMI}$ to each neutrophil reaction.

Forty-microliter (55 and 80 minutes p.i.) or $120-\mu 1$ (200 minutes p.i.) aliquots were taken from each neutrophil reaction, diluted into $500 \mu \mathrm{l}$ RPMI (in a sterile 0.6-ml Eppendorf tube), and centrifuged at 15,000 $\mathrm{g}$ for 3 minutes at room temperature. After carefully removing the supernatant, pure water $(200 \mu \mathrm{l})$ was added and pipetted vigorously 10 times to promote host cell lysis and release intracellular bacteria. The entire $200-\mu l$ cell lysate was immediately plated onto THA using the spread-plate technique $(30 \mathrm{ml}$ agar plates to further dilute out any remaining gentamicin), and CFUs were enumerated following overnight incubation at $37^{\circ} \mathrm{C}$. A similar assay was performed using human monocytes.

ChIP. The ChIP Assay Kit (Upstate Biotechnology) was used, and chromatin was prepared for immunoprecipitation as instructed by the manufacturer. The sonicated chromatin was immunoprecipitated with either $5 \mu \mathrm{g}$ anti-acetylated histone $\mathrm{H} 3$ antibody or normal rabbit IgG antibody as negative control (Upstate Biotechnology). Immunoprecipitated DNA was subsequently analyzed by PCR using primers specific for the CEBPE promoter region spanning the genomic region -797 to -518 base pairs upstream of the transcriptional start site (position: 21578530; Promoter ID 12948) that was localized by our group (10); input chromatin was analyzed for $\beta$-actin mRNA as a positive control. The optimal reaction conditions for PCR were determined for each primer pair. Primers were denatured at $95^{\circ} \mathrm{C}$ for $1 \mathrm{~min}$ ute and annealed at $60^{\circ} \mathrm{C}$ for 1 minute, followed by elongation at $72^{\circ} \mathrm{C}$ for 1 minute; each product was amplified 35 cycles. PCR products were analyzed by $2.5 \%$ agarose/ethidium bromide gel electrophoresis. Densitometry of all agarose gels was performed using Quantity One software 4.6.3 (BioRad) to quantify fold changes. The primers used for ChIP analysis were as follows: human CEBPE, 5'-GCTTTGGCCAAGCCCAGGGA-3' (forward), 5'-TGCTGGGCTCCACCTACCCC-3' (reverse); human ACTB, 5'-CTCCTCGGGAGCCACACGCA-3' (forward), 5'-TAGGGGAGCTGGCTGGGTGG-3' (reverse); murine Cebpe, 5'-TGAGGCTGCAGCTTGCCTGG-3' (forward), 5'-ACCAAGCTACCCCTGGCCCT-3' (reverse); murine Actb, 5' -ACCTGTTACTTTGGGAGTGGCAAGC-3' (forward), 5'-GTCGTCCCAGTTGGTAACAATGCC-3' (reverse).

Western blot and immunoprecipitation. Whole-cell extracts were produced by lysing cells $\left(10^{7}\right)$ with $100 \mu$ l denaturing RIPA buffer $(50 \mathrm{mM}$ Tris $\mathrm{HCl}$, $\mathrm{pH} 8 ; 150 \mathrm{mM} \mathrm{NaCl} ; 1 \% \mathrm{NP}-40 ; 0.5 \%$ sodium deoxycholate; $0.1 \% \mathrm{SDS}$ ) added with a protease inhibitor cocktail (Roche Molecular Biochemicals) on the day of extraction. Extracts were stored at $-80^{\circ} \mathrm{C}$ until use.

For Western blot, protein lysates were boiled in Laemmli sample buffer (Bio-Rad), resolved on $4 \%$ to $15 \%$ gradient SDS-PAGE gels, and transferred to nitrocellulose membranes (Sigma-Aldrich). Immunoblots were probed with anti-C/EBPE antibody (Santa Cruz Biotechnology Inc.), antiLTF antibody (Abcam), anti-CAMP antibody (Abcam), anti-gp91phox antibody (NOX2; Santa Cruz Biotechnology Inc.), and anti-lipocalin-2 antibody (NGAL; R\&D Systems) and developed using the enhanced chemiluminescence kit (Pierce). ACTB (Sigma-Aldrich) was used as a control. Western blot data are representative of 1 out of 3 independently performed experiments. Densitometry of all blots was performed using Quantity One software 4.6.3 (Bio-Rad) to quantify fold changes.

We used an anti-acetyl-lysine antibody (ab21623; Abcam) for immunoprecipitation according to the manufacturer's protocol. The input of the protein lysates was used as a loading control.

Statistics. We used 2-tailed unpaired Student's $t$ test to compare 2 independent groups when using ex vivo data; nonparametric Mann-Whitney $U$ test was applied for the independent comparison of the murine in vivo CFU data. One-way ANOVA was used for the comparison of more than 2 independent groups, and 2-way ANOVA, in combination with Bonferroni as post-hoc test, was used to compare murine body weight or lesion size data sets obtained over time. Paired Student's $t$ test was used for the comparison of human blood samples treated with either NAM or PBS. We deemed a $P$ value below 0.05 as significant. GraphPad Prism was used for analyses.

Study approval. All animal experiments were approved by the Cedars-Sinai Medical Center Institutional Animal Care and Use Committee and performed using accepted veterinary standards. Peripheral blood was obtained from healthy adult donors by the General Clinical Research Center at Cedars-Sinai Medical Center. All subjects provided written informed consent. Experimentation using human blood were approved by the Cedars- 
Sinai Medical Center Institutional Review Board and Office of Research Compliance. Experimental protocols were approved by the Cedars-Sinai Medical Center Biosafety Committee.

\section{Acknowledgments}

This work was supported by a Burroughs-Wellcome Career Award (to G.Y. Liu); grants from the US NIH to G.Y. Liu (R01 AI074832), H.P. Koeffler (R01 CA026038-30; U54 CA143930-01), and A.F. Gombart (R01 AI065604-05); and the A*STAR Investigator grant (to H.P. Koeffler). N.H. Thoennissen was supported by the Deutsche Forschungsgemeinschaft, Bonn, Germany (TH 1438/1-1). The funders had no role in study design, data collection and analysis, decision to publish, or preparation of the manuscript.
Received for publication November 22, 2011, and accepted in revised form July $5,2012$.

Address correspondence to: George Y. Liu or Pierre Kyme, Division of Pediatric Infectious Diseases and Immunobiology Research Institute, Cedars-Sinai Medical Center, 8700 Beverly Blvd., Los Angeles, California 90048, USA. Phone: 310.423.4471; Fax: 310.423.8284; E-mail: george.liu@cshs.org (G.Y. Liu); pierre.kyme@cshs.org (P. Kyme). Or to: Nils H. Thoennissen, Department of Medicine A, Hematology, Oncology and Pneumology, University of Muenster, Muenster, Germany. Phone: 49.0251.83.47604; Fax: 49.0251.83.47599; E-mail: nils.thoennissen@ukmuenster.de.
1. Lowy FD. Staphylococcus aureus infections. NEngl JMed. 1998;339(8):520-532.

2. Miller LG, Kaplan SL. Staphylococcus aureus: a community pathogen. Infect Dis Clin North Am. 2009;23(1):35-52.

3. Deleo FR, Otto M, Kreiswirth BN, Chambers HF. Community-associated meticillin-resistant Staphylococcus aureus. Lancet. 2010;375(9725):1557-1568.

4. Liu GY, et al. Staphylococcus aureus golden pigment impairs neutrophil killing and promotes virulence through its antioxidant activity. $J$ Exp Med. 2005;202(2):209-215.

5. Strauss RG, Bove KE, Jones JF, Mauer AM, Fulginiti VA. An anomaly of neutrophil morphology with impaired function. $N$ Engl J Med. 1974; 290(9):478-484

6. Komiyama A, Morosawa H, Nakahata T, Miyagawa Y, Akabane T. Abnormal neutrophil maturation in a neutrophil defect with morphologic abnormality and impaired function. J Pediatr. 1979;94(1):19-25.

7. Ambruso DR, et al. Defective bactericidal activity and absence of specific granules in neutrophils from a patient with recurrent bacterial infections. J Clin Immunol. 1984;4(1):23-30.

8. Shiohara M, et al. Phenotypic and functional alterations of peripheral blood monocytes in neutrophil-specific granule deficiency. J Leukoc Biol. 2004;75(2):190-197.

9. Liu CI, et al. A cholesterol biosynthesis inhibitor blocks Staphylococcus aureus virulence. Science. 2008;319(5868):1391-1394.

10. Chumakov AM, et al. Cloning of the novel human myeloid-cell-specific C/EBP-epsilon transcription factor. Mol Cell Biol. 1997;17(3):1375-1386.

11. Yamanaka R, et al. CCAAT/enhancer binding protein epsilon is preferentially up-regulated during granulocytic differentiation and its functional versatility is determined by alternative use of promoters and differential splicing. Proc Natl Acad SciUS A. 1997;94(12):6462-6467.

12. Yamanaka R, et al. Impaired granulopoiesis, myelodysplasia, and early lethality in CCAAT/enhancer binding protein e-deficient mice. Proc Natl Acad Sci US A. 1997;94(24):13187-13192.

13. Williams SC, et al. C/EBPepsilon is a myeloid-specific activator of cytokine, chemokine, and macrophage-colony-stimulating factor receptor genes. J Biol Chem. 1998;273(22):13493-13501.

14. Lekstrom-Himes J, Xanthopoulos KG. CCAAT/ enhancer binding protein epsilon is critical for effective neutrophil-mediated response to inflammatory challenge. Blood. 1999;93(9):3096-3105.

15. Verbeek W, et al. Myeloid transcription factor C/ EBPepsilon is involved in the positive regulation of lactoferrin gene expression in neutrophils. Blood. 1999;94(9):3141-3150

16. Tavor $\mathrm{S}$, et al. Macrophage functional maturation and cytokine production are impaired in C/EBPepsilon-deficient mice. Blood. 2002;99(5):1794-1801.

17. Gombart AF, et al. Aberrant expression of neutro- phil and macrophage-related genes in a murine model for human neutrophil-specific granule deficiency. J Leukoc Biol. 2005;78(5):1153-1165.

18. Lekstrom-Himes JA, Dorman SE, Kopar P, Holland SM, Gallin JI. Neutrophil-specific granule deficiency results from a novel mutation with loss of function of the transcription factor CCAAT/ enhancer binding protein epsilon. J Exp Med. 1999;189(11):1847-1852.

19. Gombart AF, et al. Neutrophil-specific granule deficiency: homozygous recessive inheritance of a frameshift mutation in the gene encoding transcription factor CCAAT/enhancer binding proteinepsilon. Blood. 2001;97(9):2561-2567.

20. Komatsuzawa $\mathrm{H}$, et al. Innate defences against methicillin-resistant Staphylococcus aureus (MRSA) infection. J Pathol. 2006;208(2):249-260.

21. Kai K, et al. Lactoferrin stimulates A Staphylococcus aureus killing activity of bovine phagocytes in the mammary gland. Microbiol Immunol. 2002;46(3):187-194.

22. Diarra MS, et al. Lactoferrin against Staphylococcus aureus Mastitis. Lactoferrin alone or in combination with penicillin $\mathrm{G}$ on bovine polymorphonuclear function and mammary epithelial cells colonisation by Staphylococcus aureus. Vet Immunol Immunopathol. 2003;95(1-2):33-42.

23. Roger $\mathrm{T}$, et al. Histone deacetylase inhibitors impair innate immune responses to Toll-like receptor agonists and to infection. Blood. 2011;117(4):1205-1217

24. Lee DY, Hayes JJ, Pruss D, Wolffe AP. A positive role for histone acetylation in transcription factor access to nucleosomal DNA. Cell. 1993;72(1):73-84.

25. Rundlett SE, et al. HDA1 and RPD3 are members of distinct yeast histone deacetylase complexes that regulate silencing and transcription. Proc Natl Acad Sci U S A. 1996;93(25):14503-14508

26. Glozak MA, Sengupta N, Zhang X, Seto E. Acetylation and deacetylation of non-histone proteins. Gene. 2005;363:15-23.

27. Kouzarides T. Acetylation: a regulatory modification to rival phosphorylation? EMBO J. 2000; 19(6):1176-1179

28. Ceseña TI, Cardinaux JR, Kwok R, Schwartz J. $\mathrm{CCAAT} /$ enhancer-binding protein (C/EBP) beta is acetylated at multiple lysines: acetylation of $\mathrm{C} /$ EBPbeta at lysine 39 modulates its ability to activate transcription. J Biol Chem. 2007;282(2):956-967.

29. Bitterman KJ, Anderson RM, Cohen HY, LatorreEsteves M, Sinclair DA. Inhibition of silencing and accelerated aging by nicotinamide, a putative negative regulator of yeast sir2 and human SIRT1.J Biol Chem. 2002;277(47):45099-45107.

30. Sauve AA, Schramm VL. Sir2 regulation by nicotinamide results from switching between base exchange and deacetylation chemistry. Biochemistry. 2003;42(31):9249-9256.

31. Jackson MD, Schmidt MT, Oppenheimer NJ, Denu JM. Mechanism of nicotinamide inhibition and transglycosidation by Sir2 histone/protein deacety- lases. J Biol Chem. 2003;278(51):50985-50998.

32. Niren NM. Pharmacologic doses of nicotinamide in the treatment of inflammatory skin conditions: a review. Cutis. 2006;77(1 suppl):11-16.

33. Smith IM, Burmeister LF. Biochemically assisted antibiotic treatment of lethal murine Staphylococcus aureus septic shock. Am J Clin Nutr. 1977;30(8):1364-1368.

34. Stehr A, et al. Effect of combining nicotinamide as a PARS-inhibitor with selective iNOS blockade during porcine endotoxemia. Intensive Care Med. 2003;29(6):995-1002.

35. Ungerstedt JS, Blomback M, Soderstrom T. Nicotinamide is a potent inhibitor of proinflammatory cytokines. Clin Exp Immunol. 2003;131(1):48-52.

36. Pellat-Deceunynck C, Wietzerbin J, Drapier JC. Nicotinamide inhibits nitric oxide synthase mRNA induction in activated macrophages. Biochem J. 1994;297(pt 1):53-58.

37. Li F, Chong ZZ, Maiese K. Navigating novel mechanisms of cellular plasticity with the NAD+ precursor and nutrient nicotinamide. Front Biosci. 2004;9:2500-2520.

38. Banasik M, Komura H, Shimoyama M, Ueda K. Specific inhibitors of poly(ADP-ribose) synthetase and mono(ADP-ribosyl)transferase. J Biol Chem. 1992;267(3):1569-1575.

39. Szabo C. Nicotinamide: a jack of all trades (but master of none?). Intensive Care Med. 2003; 29(6):863-866.

40. Crowley CL, Payne CM, Bernstein H, Bernstein $\mathrm{C}$, Roe D. The NAD+ precursors, nicotinic acid and nicotinamide protect cells against apoptosis induced by a multiple stress inducer, deoxycholate. Cell Death Differ. 2000;7(3):314-326.

41. Ziegler M. New functions of a long-known molecule. Emerging roles of NAD in cellular signaling. Eur J Biochem. 2000;267(6):1550-1564.

42. Skokowa J, et al. NAMPT is essential for the G-CSFinduced myeloid differentiation via a $\operatorname{NAD}(+)-$ sirtuin-1-dependent pathway. Nat Med. 2009; 15(2):151-158.

43. Murray MF. Nicotinamide: an oral antimicrobial agent with activity against both Mycobacterium tuberculosis and human immunodeficiency virus. Clin Infect Dis. 2003;36(4):453-460.

44. Wurtele $\mathrm{H}$, et al. Modulation of histone $\mathrm{H} 3$ lysine 56 acetylation as an antifungal therapeutic strategy. Nat Med. 2010;16(7):774-780.

45. Koren HS, Anderson SJ, Larrick JW. In vitro activation of a human macrophage-like cell line. Nature. 1979;279(5711):328-331.

46. Passmore JS, Lukey PT, Ress SR. The human macrophage cell line U937 as an in vitro model for selective evaluation of mycobacterial antigenspecific cytotoxic T-cell function. Immunology. 2001;102(2):146-156.

47. Bernier J, et al. Pharmacokinetics of nicotinamide in cancer patients treated with accelerated radiotherapy: the experience of the Co-operative Group 
of Radiotherapy of the European Organization for Research and Treatment of Cancer. Radiother Oncol. 1998;48(2):123-133.

48. Horsman MR, Høyer M, Honess DJ, Dennis IF, Overgaard J. Nicotinamide pharmacokinetics in humans and mice: a comparative assessment and the implications for radiotherapy. Radiother. Oncol. 1993;27(2):131-139.

49. Kaneko S, et al. Protecting axonal degeneration by increasing nicotinamide adenine dinucleotide levels in experimental autoimmune encephalomyelitis models. J Neurosci. 2006;26(38):9794-9804.

50. Cho JS, et al. IL-17 is essential for host defense against cutaneous Staphylococcus aureus infection in mice. J Clin Invest. 2010;120(5):1762-1773.

51. Gombart AF, et al. Low plasma level of cathelicidin antimicrobial peptide (hCAP18) predicts increased infectious disease mortality in patients undergoing hemodialysis. Clin Infect Dis. 2009;48(4):418-424.

52. Klaidman LK, Mukherjee SK, Hutchin TP, Adams JD. Nicotinamide as a precursor for $\mathrm{NAD}+$ prevents apoptosis in the mouse brain induced by tertiarybutylhydroperoxide. Neurosci. Lett. 1996;206(1):5-8.
53. Lomax KJ, et al. Selective defect in myeloid cell lactoferrin gene expression in neutrophil-specific granule deficiency. JClin Invest. 1989;83(2):514-519.

54. Spencer LT, et al. Role of human neutrophil peptides in lung inflammation associated with alpha1antitrypsin deficiency. Am J Physiol Lung Cell Mol Physiol. 2004;286(3):L514-L520.

55. Appelberg R. Neutrophils and intracellular pathogens: beyond phagocytosis and killing. Trends Microbiol. 2007;15(2):87-92.

56. Williamson EA, Williamson IK, Chumakov AM, Friedman AD, Koeffler HP. CCAAT/enhancer binding protein epsilon: changes in function upon phosphorylation by p38 MAP kinase. Blood. 2005;105(10):3841-3847.

57. Kaanders JH, et al. Administration of nicotinamide during a five- to seven-week course of radiotherapy: pharmacokinetics, tolerance, and compliance. Radiother Oncol. 1997;43(1):67-73.

58. Stratford MR, et al. Pharmacokinetics of nicotinamide and its effect on blood pressure, pulse and body temperature in normal human volunteers. Radiother Oncol. 1992;25(1):37-42.
59. Subcommittee on Vitamin Tolerance, Committee on Animal Nutrition, Board on Agriculture, and National Research Council. Vitamin Tolerance of Animals. Washington DC, USA: National Academies Press; 1987.

60. Bunce C, Wheeler L, Reed G, Musser J, Barg N. Murine model of cutaneous infection with grampositive cocci. Infect Immun. 1992;60(7):2636-2640.

61. Tseng CW, et al. Staphylococcus aureus PantonValentine leukocidin contributes to inflammation and muscle tissue injury. PLoS One. 2009;4(7):e6387.

62. Savov JD, Gavett SH, Brass DM, Costa DL, Schwartz DA. Neutrophils play a critical role in development of LPS-induced airway disease. Am J Physiol Lung Cell Mol Physiol. 2002;283(5):L952-L962.

63. Gao XP, et al. Inactivation of CD11b in a mouse transgenic model protects against sepsis-induced lung PMN infiltration and vascular injury. Physiol Genomics. 2005;21(2):230-242.

64. Park DJ, et al. CCAAT/enhancer binding protein epsilon is a potential retinoid target gene in acute promyelocytic leukemia treatment. J Clin Invest. 1999;103(10):1399-1408. 